\title{
A CONSTRUCTIVE WAY TO COMPUTE THE TARSKI NUMBER OF A GROUP
}

\author{
AKRAM YOUSOFZADEH
}

\begin{abstract}
The Tarski number of a group $G$ is the minimal number of the pieces of paradoxical decompositions of that group. Using configurations along with a matrix combinatorial property we construct paradoxical decompositions. We also compute an upper bound for the Tarski number of a given non-amenable group by counting the number of paths in a diagram associated to the group.
\end{abstract}

\section{INTRODUCTION}

Rosenblatt and Willis introduced a concept for groups to show that for an infinite discrete amenable group or a non-discrete amenable group $G$ a net of positive, normalized functions in $L^{1}(G)$ can be constructed such that this net converges weak* to invariance but does not converge strongly to invariance [8. This concept which is called configuration is also used to classify some group theoretical properties (see for example [1, 2]).

Configurations are strongly linked to the amenability of groups and by Tarski's alternative, a discrete group is non-amenable if and only if it admits a paradoxical decomposition. Therefore it is valuable to construct the paradoxical decomposition for such a group, using configurations. This problem which was originally asked by Willis is answered partially in [7]. In that paper the paradoxical decomposition was constructed under a paradoxical condition.

In the present paper we define a general matrix combinatorial property under which the paradoxical decomposition is completely constructed. We also find a new upper bound for the Tarski number of a given non-amenable group.

Notations. The following notations are used throughout this paper

- $\mathbb{N}, \mathbb{Z}$ and $\mathbb{R}$ are the sets of natural, integer and real numbers, respectively,

- $\sqcup$ is the disjoint union of sets,

- $g A=\{g a ; a \in A\}$, for a group $G, A \subseteq G$ and $g \in G$,

- $\mathcal{P}(X)$ is the power set of the set $X$,

- $|X|$ is the the cardinal number of the set $X$,

- A $(0,1)$-matrix is a matrix with entries in $\{0,1\}$.

If $v$ is a vector of real numbers and $n \in \mathbb{Z}$, by $v \geq n$ we mean that $v$ has entries equal to or greater than $n$. 


\section{Preliminaries}

2.1. Matrix theory. We use Gordan's theorem to find nontrivial nonnegative solutions to a homogenous system of linear equations with real coefficients. This theorem has also applications in linear programming [4].

Theorem 2.1. (Gordan 1873) Either a linear homogenous system of equations $A X=0$ possesses a nontrivial solution in nonnegative variables or there exists an equation, formed by taking some linear combination of equations, that all positive coefficients. That is, either there exists an $x$ such that

$$
A x=0, \quad 0 \neq x \geq 0
$$

or there exists a vector $m$ such that $m^{t} A>0$ (has positive entries).

Remark 2.2. In Theorem 2.1, if $A$ is a matrix with rational entries, then the entries of $m$ can be chosen in $\mathbb{Z}$.

The main theorem of this paper is proved under a certain condition. To clarify this condition, we need some definitions.

Let $\pi:\{1, \ldots, n\} \rightarrow\{1, \ldots, n\}$ be a permutation for the set $\{1, \ldots, n\}$. Then

$$
P_{\pi}=\left(\begin{array}{c}
e_{\pi(1)} \\
e_{\pi(2)} \\
\vdots \\
e_{\pi(n)}
\end{array}\right)
$$

is called the permutation matrix associated to $\pi$, where $e_{i}$ denotes the row vector of length $n$ with 1 in the i-th position and 0 otherwise. When the permutation matrix $P_{\pi}$ is multiplied with a matrix $M$ from left, $P_{\pi} M$ will permute the rows of $M$ by $\pi$.

$$
\text { If } P=\left(\begin{array}{c}
P_{1} \\
P_{2} \\
\vdots \\
P_{n}
\end{array}\right) \text { is a permutation matrix, by } P^{+} \text {we mean the matrix with }
$$
shifted rows, i.e.

$$
P^{+}=P_{\rho} P=\left(\begin{array}{c}
P_{2} \\
P_{3} \\
\vdots \\
P_{n} \\
P_{1}
\end{array}\right)
$$

in which $\rho$ is the cyclic permutation $\left(\begin{array}{lll}1 & 2 \ldots n\end{array}\right)$. Throughout we use the notation

$$
T=\left(\begin{array}{ccccc}
1 & 0 & 0 & \ldots & 0 \\
1 & 1 & 0 & \ldots & 0 \\
\vdots & & & & \\
1 & 1 & 1 & \ldots & 1
\end{array}\right)=\sum_{1 \leq j \leq i \leq n} E_{i j}
$$

where $E_{i j}$ is the matrix with 1 in $i j$ position and 0 otherwise. When the matrix $T$ is multiplied with a matrix $M$ from left, $\mathrm{j}$-th row of $T M$ will be the sum of $\mathrm{j}$ first rows of $M$. 
Definition 2.3. Let $\ell \in \mathbb{N}$ and $\left(\begin{array}{c}A_{1} \\ A_{2} \\ \vdots \\ A_{n}\end{array}\right)$ and $\left(\begin{array}{c}B_{1} \\ B_{2} \\ \vdots \\ B_{n}\end{array}\right)$ be two (0,1)-matrices with rows $A_{i}, B_{i}$. Let also the vector $\sum_{i=1}^{n}\left(B_{i}-A_{i}\right)$ has strictly positive entries. If there exists a permutation matrix $P_{\pi}$ such that the matrix

$$
T P_{\pi}(B-A)-P_{\pi}^{+} A
$$

has integer entries equal or greater than -1 , we say that the homogenous system of equations $(B-A) X=0$ is normal.

It is apparent that if $\sum_{i=1}^{n}\left(B_{i}-A_{i}\right)$ has strictly positive entries, then the system has no non-zero non-negative solution. Conversely, if $(B-A) X=0$ is a system of equations with no non-zero non-negative solution, then by Gordan's theorem there exists a vector $m=\left(m_{1}, \ldots, m_{n}\right)$ such that $m^{t}(B-A)$ has strictly positive entries. If we permit $B-A$ to have repeated rows and we insert the opposite of a row (exchanging the corresponding rows of $A$ and $B$ ) if necessary, then $m_{i}$ can be chosen in $\{0,1\}$. Omit $B_{i}-A_{i}$, where $m_{i}=0$ and denote the modified matrix by $B-A$ again. So, we can assume that $\sum_{i=1}^{n}\left(B_{i}-A_{i}\right)$ has strictly positive entries.

It is to be noted that there exist examples of both normal and non-normal systems of equations with no nonzero non-negative solution. We will explain the relation between normality and paradoxical decompositions in section 3 .

2.2. Non-amenable discrete groups. Let $G$ be a discrete group. Then $G$ is called amenable if it admits a finitely additive probability measure $\mu$ on the $\sigma$-algebra $\mathcal{P}(G)$ such that

$$
\mu(g A)=\mu(A), \quad(A \subseteq G, g \in G) .
$$

Definition 2.4. 10 Let $G$ be a group acting on a set $X$ and suppose $E \subseteq X$. $E$ is $G$-paradoxical (or, paradoxical with respect to $G$ ) if for some positive integers $m, n$ there are pairwise disjoint subsets $A_{1}, \ldots, A_{n}, B_{1}, \ldots, B_{m}$ of $E$ and $g_{1} \ldots, g_{n}, h_{1} \ldots, h_{m} \in G$ such that

$$
E=\bigsqcup_{i=1}^{n} g_{i} A_{i}=\bigsqcup_{j=1}^{m} h_{j} B_{i} .
$$

A group $G$ is called paradoxical if it is $G$-paradoxical, where $G$ acts on itself by left multiplication. Clearly if $G$ is a paradoxical group satisfying the above definition, then it cannot be amenable. Indeed if $\mu$ is a $G$-invariant probability measure, then

$$
\begin{aligned}
1 & =\mu(G)=\sum \mu\left(A_{i}\right)+\sum \mu\left(B_{j}\right) \\
& =\sum \mu\left(g_{i} A_{i}\right)+\sum \mu\left(h_{j} B_{j}\right)=\mu(G)+\mu(G)=2 .
\end{aligned}
$$

In fact there is the following remarkable alternative due to Alfred Tarski.

Theorem 2.5. Let $G$ be a discrete group. Exactly one of the following happens

1) $G$ is paradoxical,

2) $G$ is amenable. 
The number $\tau=n+m$ for $n$ and $m$ in (2) is called the Tarski number of that paradoxical decomposition; the minimum of all such numbers over all the possible paradoxical decompositions of $G$, is called the Tarski number of $G$ and denoted by $\tau(G)$. In the case that there is no paradoxical decomposition, we set $\tau(G)=\infty$. The Tarski number of a group is of real interest and has been estimated for some classes of groups. But it is not so easy to compute in many cases. For more details see 9 and 3 .

We draw the reader's attention to the next proposition for different types of paradoxical decompositions.

Proposition 2.6. [7, Proposition 1.2] Let $G$ be a group. Then the following statements are equivalent

1) There exist a partition $\left\{A_{1}, \ldots, A_{n}, B_{1}, \ldots, B_{m}\right\}$ of $G$ and $g_{1}, \ldots, g_{n}$ and $h_{1}, \ldots, h_{m}$ in $G$ such that $\left\{g_{i} A_{i}\right\}_{i=1}^{n}$ and $\left\{h_{j} B_{j}\right\}_{j=1}^{m}$ form partitions of $G$.

2) There exist pairwise disjoint subsets $A_{1}, \ldots, A_{n}, B_{1}, \ldots, B_{m}$ of $G$ and elements $g_{1}, \ldots, g_{n}$ and $h_{1}, \ldots, h_{m}$ in $G$ such that $\left\{g_{i} A_{i}\right\}_{i=1}^{n}$ and $\left\{h_{j} B_{j}\right\}_{j=1}^{m}$ form partitions of $G$.

3) There exist pairwise disjoint subsets $A_{1}, \ldots, A_{n}, B_{1}, \ldots, B_{m}$ of $G$ and elements $g_{1}, \ldots, g_{n}$ and $h_{1}, \ldots, h_{m}$ in $G$ such that $G=\bigcup_{i=1}^{n} g_{i} A_{i}=\bigcup_{j=1}^{m} h_{j} B_{j}$ (not necessarily pairwise disjoint).

Because of the above equivalence, $G$ admits a paradoxical decomposition, if any condition of Proposition 2.6 holds. The decomposition in condition 1 is called a complete paradoxical decomposition. By [5, Theorem A.1] if the conditions 2 or 3 are satisfied for a group $G$, then $\tau(G) \leq m+n$.

2.3. Configuration of groups. Let $G$ be a discrete group. The configurations of $G$ are defined in terms of finite generating sets and finite partitions of $G$. If $\mathfrak{g}=\left(g_{1}, \ldots, g_{n}\right)$ is a string of elements of $G$ and $\mathcal{E}=\left\{E_{1}, \ldots, E_{m}\right\}$ is a partition of $G$, a configuration corresponding to $(\mathfrak{g}, \mathcal{E})$ is an $(n+1)$-tuple $C=\left(c_{0}, \ldots, c_{n}\right)$, where $1 \leq c_{i} \leq m$ for each $i$, such that there is $x$ in $G$ with $x \in E_{c_{0}}$ and $g_{i} x \in E_{c_{i}}$ for each $1 \leq i \leq n$. The set of all configurations corresponding to the pair $(\mathfrak{g}, \mathcal{E})$ will be denoted by $\operatorname{Con}(\mathfrak{g}, \mathcal{E})$. It is shown that groups with the same set of configurations have some common properties. For example they obey the same semigroup laws and have the same Tarski numbers (see [1] and [1]).

In the case that $\mathfrak{g}=\left\{g_{1}, \ldots, g_{n}\right\}$ is a generating set for $G$, the configuration $C=\left(c_{0}, \ldots, c_{n}\right)$ may be described as a labelled tree which is a subgraph of the Cayley graph of the finitely generated group $G$ and configuration set $\operatorname{Con}(\mathfrak{g}, \mathcal{E})$ is a set of rooted trees having height 1 . In last section of the paper we assign a new graph to $G$ that depends on the pair $(\mathfrak{g}, \mathcal{E})$.

If $(\mathfrak{g}, \mathcal{E})$ is as above and for each $C \in \operatorname{Con}(\mathfrak{g}, \mathcal{E})$

$$
x_{0}(C)=E_{c_{0}} \cap\left(\cap_{j=1}^{n} g_{j}^{-1} E_{c_{j}}\right) \quad \text { and } \quad x_{j}(C)=g_{j} x_{0}(C) \text {, }
$$

then it is seen that for any $0 \leq j \leq n,\left\{x_{j}(C) ; C \in C o n(\mathfrak{g}, \mathcal{E})\right\}$ is a partition for $G$. Let $C \in \operatorname{Con}(\mathfrak{g}, \mathcal{E})$ and $f \in \ell^{1}(G)$. Define $f_{C}=\sum_{x \in x_{0}(C)} f(x)$. Then we have (see [8])

if and only if

$$
\left\langle f-g_{j} f, \chi_{E_{i}}\right\rangle=0, \quad(1 \leq j \leq n, 1 \leq i \leq m)
$$

$$
\sum_{x_{0}(C) \subseteq E_{i}} f_{C}=\sum_{x_{j}(C) \subseteq E_{i}} f_{C} \quad(1 \leq j \leq n, 1 \leq i \leq m) .
$$


For each pair $(\mathfrak{g}, \mathcal{E})$ for $G$, the system of equations

$$
\sum_{x_{j}(C) \subseteq E_{i}} f_{C}=\sum_{x_{k}(C) \subseteq E_{i}} f_{C}, \quad(1 \leq i \leq m, 0 \leq j, k \leq n)
$$

with variables $f_{C}, C \in C o n(\mathfrak{g}, \mathcal{E})$ is called the system of configuration equations corresponding to $(\mathfrak{g}, \mathcal{E})$ and is denoted by $E q(\mathfrak{g}, \mathcal{E})$. By a normalized solution to this system, we mean a solution $\left(f_{C}\right)_{C}$ such that for each $C, f_{C} \geq 0$ and $\sum_{C} f_{C}=1$. A group $G$ is amenable if and only if there is a net $\left(f_{\alpha}\right)_{\alpha}$ of positive functions in $L^{1}(G)$ with $\int f_{\alpha}=1$ which is weak* convergence to invariance, that is for any $F \in L^{\infty}(G)$ and any $g \in G$

$$
\lim _{\alpha}\left\langle f_{\alpha}-{ }_{g} f_{\alpha}, F\right\rangle=0
$$

(see [6]). Using this fact, Rosenblatt and Willis proved the following theorem

Theorem 2.7. 8, Proposition 2.4] There is a normalized solution of every possible instance of the configuration equations if and only if $G$ is amenable.

We will apply this theorem to find paradoxical decompositions.

\section{MAIN TheOREM}

Throughout this section by $G, \mathfrak{g}=\left(g_{1}, \ldots, g_{n}\right)$ and $\mathcal{E}=\left\{E_{1}, \ldots, E_{m}\right\}$ we mean a group, a finite string of elements of $G$ and a finite partition for $G$, respectively. If $D$ is a subset of $\operatorname{Con}(\mathfrak{g}, \mathcal{E})$, we use the following notation

$$
\tilde{D}:=\bigsqcup_{C \in D} x_{0}(C) .
$$

In particular $\operatorname{Con} \tilde{(\mathfrak{g}}, \mathcal{E})=G$. Clearly $D_{1}, D_{2} \subseteq \operatorname{Con}(\mathfrak{g}, \mathcal{E})$ are disjoint if and only if $\tilde{D}_{1}$ and $\tilde{D}_{2}$ are. The configuration equation $\sum_{x_{j}(C) \subseteq E_{i}} f_{C}=\sum_{x_{k}(C) \subseteq E_{i}} f_{C}$ is written in the form $\mathfrak{a} X=\mathfrak{b} X$, where $\operatorname{Con}(\mathfrak{g}, \mathcal{E})=\left\{C_{1}, \ldots, C_{\ell}\right\}$,

$$
X=\left(\begin{array}{c}
f_{C_{1}} \\
f_{C_{2}} \\
\vdots \\
f_{C_{\ell}}
\end{array}\right)
$$

$\mathfrak{a}$ is the coefficient vector of the left hand side and $\mathfrak{b}$ is the coefficient vector of the right hand side of the equation.

Theorem 3.1. If a subsystem of $E q(\mathfrak{g}, \mathcal{E})$ is normal, then $G$ is non-amenable and a paradoxical decomposition of $G$ can be written in terms of $\mathfrak{g}$ and $\mathcal{E}$.

Proof. Let $V=\left(\begin{array}{c}V_{1} \\ V_{2} \\ \vdots \\ V_{p}\end{array}\right)$ and $W=\left(\begin{array}{c}W_{1} \\ W_{2} \\ \vdots \\ W_{p}\end{array}\right)$ be $(0,1)$-matrices in $M_{p \times \ell}(\mathbb{R})$ such that $(W-V) X=0$ is the normal subsystem of $E q(\mathfrak{g}, \mathcal{E})$ satisfying (1) for a permutation matrix $P_{\pi}$. Write

$$
V_{t}=\left(V_{t}(C)\right)_{C \in \operatorname{Con}(\mathfrak{g}, \mathcal{E})} \quad \text { and } \quad W_{t}=\left(W_{t}(C)\right)_{C \in \operatorname{Con}(\mathfrak{g}, \mathcal{E})}, \quad(1 \leq t \leq p) .
$$


Then by the normality of $(W-V) X=0$, for each $C \in C o n(\mathfrak{g}, \mathcal{E})$

$$
\alpha_{C}:=\sum_{t=1}^{p} W_{t}(C)-\sum_{t=1}^{p} V_{t}(C)>0 .
$$

We consider only the case that $P_{\pi}=I$, the identity matrix. Otherwise defining $V_{t}^{*}=V_{\pi(t)}$ and $W_{t}^{*}=W_{\pi(t)}$ we see that

$$
T P_{\pi}(W-V)-P_{\pi}^{+} V=T\left(W^{*}-V^{*}\right)-V^{*+}
$$

and the proof is carried out in a similar way. Therefore throughout the proof we assume

$$
\begin{aligned}
& W_{1}(C)-\left(V_{1}(C)+V_{2}(C)\right) \geq-1, \\
&\left(W_{1}(C)+W_{2}(C)\right)-\left(V_{1}(C)+V_{2}(C)+V_{3}(C)\right) \geq-1, \vdots \\
&\left.\left(\sum_{t=1}^{p-1} W_{t}(C)\right)-\sum_{t=1}^{p} V_{t}(C)\right) \geq-1 .
\end{aligned}
$$

Suppose that $\left(i_{1}, i_{2}, \ldots, i_{p}\right),\left(j_{i_{1}}, j_{i_{2}}, \ldots, j_{i_{p}}\right)$ and $\left(k_{i_{1}}, k_{i_{2}}, \ldots, k_{i_{p}}\right)$ are strings such that $i_{s} \in\{1, \ldots, m\}$ and $j_{i_{s}}, k_{i_{s}} \in\{0,1, \ldots, n\}$ and the modified system is

$$
\sum_{x_{j_{i_{t}}}(C) \subseteq E_{i_{t}}} f_{C}=\sum_{x_{k_{i_{t}}}(C) \subseteq E_{i_{t}}} f_{C}, \quad(1 \leq t \leq p) .
$$

Note that the strings are used instead of subsets since the repetition is not excluded for the equations. For convenience we use the following notations for $1 \leq t \leq p$

$$
A_{t}=\left\{C ; \quad x_{k_{i_{t}}}(C) \subseteq E_{i_{t}}\right\} \text { and } B_{t}=\left\{C ; \quad x_{j_{i_{t}}}(C) \subseteq E_{i_{t}}\right\}, \quad(1 \leq t \leq p) .
$$

In other words, the system can be written as

$$
\sum_{C \in A_{t}} f_{C}=\sum_{C \in B_{t}} f_{C}, \quad(1 \leq t \leq p) .
$$

It is not difficult to see that for $1 \leq t \leq p$

$$
\dot{g}_{t} \bigsqcup_{C^{*} \in A_{t}} x_{0}\left(C^{*}\right)=\bigsqcup_{C \in B_{t}} x_{0}(C)
$$

where $\dot{g}_{t}=g_{k_{i_{t}}}^{-1} g_{j_{i_{t}}}$. For $C \in B_{t}$ and $C^{*} \in A_{t}$ put

$$
E_{t}\left(C^{*}, C\right)=x_{0}\left(C^{*}\right) \cap \dot{g}_{t}^{-1} x_{0}(C) .
$$

Then we have by (5)

$$
x_{0}(C)=\bigsqcup_{C^{*} \in A_{t}} \dot{g}_{t} E_{t}\left(C^{*}, C\right), \quad\left(C \in B_{t}\right) .
$$

Case 1. The sets $A_{t}$ are pairwise disjoint. According to (3) and (4), for each $C \in \mathfrak{A}:=\bigsqcup_{t=1}^{p} A_{t}$ there exist at least two numbers $1 \leq t_{1}^{C}, t_{2}^{C} \leq p$ such that 
$C \in B_{t_{1}^{C}}$ and $C \in B_{t_{2}^{C}}$. Also for each $C \in \mathfrak{B}:=\operatorname{Con}(\mathfrak{g}, \mathcal{E}) \backslash \mathfrak{A}$ there exists at least one $1 \leq t^{C} \leq p$ such that $C \in B_{t^{C}}$. This fact together with (6) implies that

$$
x_{0}(C)=\bigsqcup_{C^{*} \in A_{t_{1}^{C}}} \dot{g}_{t_{1}^{C}} E_{t_{1}^{C}}\left(C^{*}, C\right)=\bigsqcup_{C^{*} \in A_{t_{2}^{C}}} \dot{g}_{t_{2}^{C}} E_{t_{2}^{C}}\left(C^{*}, C\right), \quad(C \in \mathfrak{A})
$$

and

$$
x_{0}(C)=\bigsqcup_{C^{*} \in A_{t} C} \dot{g}_{t^{C}} E_{t^{C}}\left(C^{*}, C\right), \quad(C \in \mathfrak{B}) .
$$

Consequently the fact that

$$
G=\left[\bigsqcup_{C \in \mathfrak{A}} x_{0}(C)\right] \bigsqcup\left[\bigsqcup_{C \in \mathfrak{B}} x_{0}(C)\right]
$$

necessitates the following two equations

$$
G=\left[\bigsqcup_{C \in \mathfrak{A}} \bigsqcup_{C^{*} \in A_{t_{1}^{C}}} \dot{g}_{t_{1}^{C}} E_{t_{1}^{C}}\left(C^{*}, C\right)\right] \bigsqcup\left[\bigsqcup_{C \in \mathfrak{B}} x_{0}(C)\right]
$$

and

$$
G=\left[\bigsqcup_{C \in \mathfrak{A}} \bigsqcup_{C^{*} \in A_{t_{2}^{C}}} \dot{g}_{t_{2}^{C}} E_{t_{2}^{C}}\left(C^{*}, C\right)\right] \bigsqcup\left[\bigsqcup_{C \in \mathfrak{B}} \bigsqcup_{C^{*} \in A_{t^{C}}} \dot{g}_{t^{C}} E_{t^{C}}\left(C^{*}, C\right)\right]
$$

We emphasize that all the sets in the following families are pairwise disjoint

(1) $x_{0}(C)$, with $C \in \mathfrak{B}$,

(2) $E_{t^{C}}\left(C^{*}, C\right)$, with $C \in \mathfrak{B}, C^{*} \in A_{t^{C}}$,

(3) $E_{t_{1}^{C}}\left(C^{*}, C\right)$, with $C \in \mathfrak{A}, C^{*} \in A_{t_{1}^{C}}$,

(4) $E_{t_{2}^{C}}\left(C^{*}, C\right)$, with $C \in \mathfrak{A}, C^{*} \in A_{t_{2}^{C}}$.

We know that $\mathfrak{A} \cap \mathfrak{B}=\emptyset$ and $A_{i} \cap A_{j}=\emptyset$ for $i \neq j$. On the other hand for $C^{*} \in A_{t}, E_{t}\left(C^{*}, C\right) \subseteq x_{0}\left(C^{*}\right) \subseteq \tilde{A}_{t} \subseteq \tilde{\mathfrak{A}}$. Therefore the sets of types (2), (3) and (4) are all disjoint from the sets of type (1). Since $t_{1}^{C} \neq t_{2}^{C}, E_{t_{1}^{C}}\left(C^{*}, C\right) \subseteq A_{t_{1}^{C}}$ and $E_{t_{2}^{C}}\left(C^{*}, C\right) \subseteq A_{t_{2}^{C}}$ and $A_{t_{1}^{C}} \cap A_{t_{2}^{C}}=\emptyset$, we have $E_{t_{1}^{C}}\left(C^{*}, C\right) \cap E_{t_{2}^{C}}\left(C^{*}, C\right)=\emptyset$. Now let $i \in\{1,2\}$ and $t_{i}^{C^{\prime}}=t^{C}$, for $C \in \mathfrak{B}$ and $C^{\prime} \in \mathfrak{A}$. Let $C^{*} \in A_{t^{C}}$ and $C^{\prime *} \in A_{t_{i}^{C^{\prime}}}$. Applying $\mathfrak{A} \cap \mathfrak{B}=\emptyset$ once again, we see $g_{t^{C}}^{-1} x_{0}(C) \cap g_{t_{i}^{\prime}}^{-1} x_{0}\left(C^{\prime}\right)=\emptyset$. Thus $E_{t_{i}^{C^{\prime}}}\left(C^{\prime *}, C^{\prime}\right) \cap E_{t^{C}}\left(C^{*}, C\right)=\emptyset$. Therefore all the sets of types $(3)$ and (4) are disjoint from the sets of type (2). It remains to show that for distinct configurations $C, D \in \mathfrak{A}, i, j \in\{1,2\}, C^{*} \in A_{t_{i}^{C}}$ and $D^{*} \in A_{t_{j}^{D}}$ one has $E_{t_{i}^{C}}\left(C^{*}, C\right) \cap E_{t_{j}^{D}}\left(D^{*}, D\right)=$ $\emptyset$. But it is clear since if $t_{i}^{C} \neq t_{j}^{D}$, then

$$
E_{t_{i}^{C}}\left(C^{*}, C\right) \cap E_{t_{j}^{D}}\left(D^{*}, D\right) \subseteq x_{0}\left(C^{*}\right) \cap x_{0}\left(D^{*}\right) \subseteq \tilde{A}_{t_{i}^{C}} \cap \tilde{A}_{t_{j}^{D}}=\emptyset
$$

and if $t_{i}^{C}=t_{j}^{D}$, then

$$
E_{t_{i}^{C}}\left(C^{*}, C\right) \cap E_{t_{j}^{D}}\left(D^{*}, D\right) \subseteq \dot{g}_{t_{i}^{C}}^{-1}\left(x_{0}(C) \cap x_{0}(D)\right)=\dot{g}_{t_{i}^{C}}^{-1}(\emptyset)=\emptyset .
$$

Consequently the equations (77) and (8) form a paradoxical decomposition of $G$.

Case 2. The sets $A_{t}$ are not pairwise disjoint. The procedure in case 1 does not work here because the sets in a paradoxical decomposition must be disjoint. 
In the sequel we shall replace the sets $A_{1}, \ldots, A_{p}$ with new sets $P_{1}, \ldots, P_{p}$, which are disjoint and then a paradoxical decomposition with respect to a partition of $\bigsqcup_{i=1}^{p} \tilde{P}_{i}$ will be given.

For $C \in \operatorname{Con}(\mathfrak{g}, \mathcal{E})$ if $t_{0}$ is the least number in $\{1, \ldots, p\}$ such that $V_{t_{0}}(C) \neq 0$, define $V_{t_{0}}^{\prime}(C)=1$ and for $t \neq t_{0}$ put $V_{t}^{\prime}(C)=0$. Then

$$
\sum_{t=1}^{p} V_{t}^{\prime}(C)= \begin{cases}1, & \text { if } \sum_{t=1}^{p} V_{t}(C)>0 \\ 0, & \text { otherwise. }\end{cases}
$$

We need a counter to compute the number of appearance of a configuration in $V_{1}, \ldots, V_{p}$ and $W_{1}, \ldots, W_{p}$. To achieve this purpose define

$$
m_{C}^{0}= \begin{cases}\left(\sum_{t=1}^{p} V_{t}(C)\right)-1, & \text { if } \sum_{t=1}^{p} V_{t}(C)>0 \\ 0, & \text { otherwise }\end{cases}
$$

and for $1 \leq t \leq p$

$$
m_{C}^{t}= \begin{cases}m_{C}^{t-1}-1, & \text { if } W_{t}(C)=1, m_{C}^{t-1}>0 \\ m_{C}^{t-1}, & \text { otherwise. }\end{cases}
$$

Consequently by (10) we have for $C \in \operatorname{Con}(\mathfrak{g}, \mathcal{E})$

$$
\sum_{t=1}^{p} V_{t}(C)-\sum_{t=1}^{p} V_{t}^{\prime}(C)=m_{C}^{0}
$$

Consider the vectors $\left(W_{t}^{\prime}(C)\right)_{\operatorname{Con}(\mathfrak{g}, \mathcal{E})}, 1 \leq t \leq p$ with the following definition

$$
W_{t}^{\prime}(C)= \begin{cases}W_{t}(C)-1, & \text { if } W_{t}(C)=1, m_{C}^{t-1}>0 \\ W_{t}(C), & \text { otherwise. }\end{cases}
$$

It is seen by (11) that for every $C \in \operatorname{Con}(\mathfrak{g}, \mathcal{E})$

$$
\begin{aligned}
\sum_{t=1}^{p} W_{t}(C)-\sum_{t=1}^{p} W_{t}^{\prime}(C) & =\sum_{t=1}^{p}\left[W_{t}(C)-W_{t}^{\prime}(C)\right] \\
& =\sum_{t=1}^{p}\left[m_{C}^{t-1}-m_{C}^{t}\right] \\
& =m_{C}^{0}-m_{C}^{p} \\
& =m_{C}^{0} .
\end{aligned}
$$

Thus by (12) and (31)

$$
\sum_{t=1}^{p} W_{t}^{\prime}(C)-\sum_{t=1}^{p} V_{t}^{\prime}(C)=\sum_{t=1}^{p} W_{t}(C)-\sum_{t=1}^{p} V_{t}(C)=\alpha_{C} .
$$

Put

$$
P_{t}:=\left\{C: \quad V_{t}^{\prime}(C) \neq 0\right\} \text { and } Q_{t}:=\left\{C: \quad W_{t}^{\prime}(C) \neq 0\right\} .
$$

Then by (9) it is evident that $P_{1}, \ldots, P_{p}$ are pairwise disjoint. It is also clear for $1 \leq t \leq p$ that $P_{t} \subseteq A_{t}, Q_{t} \subseteq B_{t}$ and $A_{t} \backslash P_{t}=\bigcup_{j=1}^{t-1}\left(A_{t} \cap A_{j}\right)$. Let $1 \leq t<j \leq p$ and define

$$
D_{t}^{j}=\left[\left(B_{t} \backslash \bigsqcup_{s=t+1}^{j-1} D_{t}^{s}\right) \cap\left(A_{j} \backslash P_{j}\right)\right] \backslash \bigsqcup_{k=1}^{t-1} D_{k}^{j},
$$


using the conventions $\bigcup_{l=l_{1}}^{l_{2}} E_{l}=\emptyset$, when $0 \leq l_{2}<l_{1}$. Obviously

$$
D_{t}^{j} \cap D_{t}^{j^{\prime}}=\emptyset \quad \text { and } \quad D_{t}^{j} \cap D_{t^{\prime}}^{j}=\emptyset, \quad\left(t \neq t^{\prime}, j \neq j^{\prime}\right) .
$$

Next for $1 \leq t \leq p-1$ we prove that

$$
Q_{t}=B_{t} \backslash\left(\bigsqcup_{j=t+1}^{p} D_{t}^{j}\right)
$$

To see this, let $C \in Q_{t}$. Then $C \in B_{t}$ and $W_{t}^{\prime}(C)=1$, which means that $m_{C}^{t-1}=0$. If $m_{C}^{0}=0$, then for each $2 \leq j \leq p, C \notin A_{j} \backslash P_{j}$. In particular $C \notin D_{t}^{j}$, for every $t+1 \leq j \leq p$. Assume that $m_{C}^{t-1}=k>0$. Then by (10) there are exactly $k+1$ numbers $j_{0}<j_{1}<\cdots<j_{k}$ such that $C \in \cap_{i=0}^{k} A_{j_{i}}$ or equivalently

$$
C \in A_{j_{i}} \backslash P_{j_{i}}, \quad(1 \leq i \leq k) .
$$

This along with the normality condition $\left(\sum_{j=1}^{t-1} W_{j}-\sum_{j=1}^{t} V_{j} \geq-1\right)$ implies that there exist natural numbers $t_{1}<t_{2}<\cdots<t_{k}<t$ such that $C \in B_{t_{i}},(1 \leq i \leq k)$, where $t_{1}$ is the smallest number with this property. Therefore by (14) and the sentence after that $C \in \bigcap_{i=1}^{k} D_{t_{i}}^{j_{i}}$. So, we have $C \notin \bigsqcup_{j=t+1}^{p} D_{t}^{j}$. The converse is obtained by a similar argument. We continue the proof of the theorem in the following four steps.

Step 1. By the normality condition if $1 \leq t \leq p$, then $\sum_{j=1}^{t-1} W_{j}-\sum_{j=1}^{t} V_{j} \geq-1$. This means $\left(A_{t} \backslash P_{t}\right) \subseteq \cup_{j=1}^{t-1} B_{j}$. We shall prove that for $1 \leq t \leq p$

$$
A_{t} \backslash P_{t}=\bigsqcup_{s=1}^{t-1} D_{s}^{t}
$$

Firstly we show that

$$
A_{t} \backslash P_{t} \subseteq \bigcup_{s=1}^{t-1}\left[\left(B_{s} \backslash \cup_{j=s+1}^{t-1} D_{s}^{j}\right) \backslash \bigsqcup_{k=1}^{s-1} D_{k}^{t}\right] .
$$

Let $C \in A_{t} \backslash P_{t}$ and pick the smallest natural number $t_{0}<t$ such that $C \in B_{t_{0}}$ (it is possible by the normality condition). If for each $t_{0}<j<t$ and $1 \leq k<t_{0}$, $C \notin D_{t_{0}}^{j}$ and $C \notin D_{k}^{j}$, we are done. If $C \in \bigsqcup_{k=1}^{t_{0}-1} D_{k}^{t}$, then $C \in \bigsqcup_{k=1}^{t-1} D_{k}^{t}$ because $t_{0}<t$. Assume that for some $t_{0}<j<t, C \in D_{t_{0}}^{j}$. Then by (14) $C \in A_{j} \backslash P_{j}$. By the definition of $P_{j}$, this means that there exists at least one natural number $s<j$ such that $C \in A_{j} \cap A_{s}$ but on the other side $C \in A_{t}$. Consequently $C \in A_{j} \cap A_{s} \cap A_{t}$. Since by the normality condition $\sum_{j=1}^{t-1} W_{j}-\sum_{j=1}^{t} V_{j} \geq-1$, there are at least two sets of the form $B_{i}$ containing $C$. Accordingly there exists the smallest positive integer $t_{0}^{\prime}<t$ such that $C \in B_{t_{0}^{\prime}}$. This process is obviously finite and therefore we achieve the purpose. Hence

$$
A_{t} \backslash P_{t}=\left(A_{t} \backslash P_{t}\right) \cap \bigcup_{s=1}^{t-1}\left[\left(B_{s} \backslash \cup_{j=s+1}^{t-1} D_{s}^{j}\right) \backslash \bigsqcup_{k=1}^{s-1} D_{k}^{t}\right]=\bigsqcup_{s=1}^{t-1} D_{s}^{t} .
$$

Step 2. We show by induction that for all $j \geq 1$ there exists a family $\left\{\mathcal{A}_{\sigma_{j}}, \quad \sigma_{j} \in\right.$ $\left.\Lambda_{j}\right\}$ with cardinality $2^{j-1}-1$ of disjoint subsets (possibly the empty sets) of $\bigsqcup_{t=1}^{j-1} \tilde{P}_{t}$ 
and the members $\left\{g_{\sigma_{j}}, \quad \sigma_{j} \in \Lambda_{j}\right\}$ of $G$ such that

$$
\tilde{A}_{j} \backslash \tilde{P}_{j}=\bigsqcup_{\sigma_{j} \in \Lambda_{j}} g_{\sigma_{j}} \mathcal{A}_{\sigma_{j}} .
$$

Since $\tilde{A}_{1} \backslash \tilde{P}_{1}=\emptyset$, it is natural to set $\mathcal{A}_{\sigma_{1}}=\emptyset$ and $g_{\sigma_{1}}=e$, the identity element of $G$. For $j=2$, we have $\tilde{A}_{2} \backslash \tilde{P}_{2}=\tilde{A}_{1} \cap \tilde{A}_{2} \subseteq \tilde{B}_{1}=\dot{g}_{1} \tilde{A}_{1}$. Hence $\tilde{A}_{2} \backslash \tilde{P}_{2}=\dot{g}_{1}\left(\dot{g}_{1}^{-1} \tilde{D}_{1}^{2}\right)$. Set $\sigma_{2}=1, \Lambda_{2}=\left\{\sigma_{2}\right\}$ and $g_{\sigma_{2}}=\dot{g}_{1}$. Note that $\left|\Lambda_{2}\right|=1=2^{2-1}-1$. Now let for $1 \leq t \leq j$

$$
\tilde{A}_{t} \backslash \tilde{P}_{t}=\bigsqcup_{\sigma_{t} \in \Lambda_{t}} g_{\sigma_{t}} \mathcal{A}_{\sigma_{t}} \quad \text { and } \quad\left|\Lambda_{t}\right|=2^{t-1}-1 .
$$

By (16)

$$
\begin{aligned}
\tilde{A}_{j+1} \backslash \tilde{P}_{j+1} & =\bigsqcup_{t=1}^{j} \tilde{D}_{t}^{j+1} \\
& =\bigsqcup_{t=1}^{j} \dot{g}_{t}\left(\dot{g}_{t}^{-1} \tilde{D}_{t}^{j+1}\right) \\
& =\bigsqcup_{t=1}^{j} \dot{g}_{t}\left(\left[\dot{g}_{t}^{-1} \tilde{D}_{t}^{j+1} \cap \tilde{P}_{t}\right] \bigsqcup\left[\dot{g}_{t}^{-1} \tilde{D}_{t}^{j+1} \cap\left(\tilde{A}_{t} \backslash \tilde{P}_{t}\right)\right]\right) \\
& =\bigsqcup_{t=1}^{j} \dot{g}_{t}\left(\left[\dot{g}_{t}^{-1} \tilde{D}_{t}^{j+1} \cap \tilde{P}_{t}\right] \bigsqcup\left[\dot{g}_{t}^{-1} \tilde{D}_{t}^{j+1} \cap\left(\bigsqcup_{\sigma_{t} \in \Lambda_{t}} g_{\sigma_{t}} \mathcal{A}_{\sigma_{t}}\right)\right]\right) \\
& =\left(\bigsqcup_{t=1}^{j} \dot{g}_{t}\left(\dot{g}_{t}^{-1} \tilde{D}_{t}^{j+1} \cap \tilde{P}_{t}\right)\right) \bigsqcup\left(\bigsqcup_{t=2}^{j} \bigsqcup_{\sigma_{t} \in \Lambda_{t}} \dot{g}_{t} g_{\sigma_{t}}\left[g_{\sigma_{t}}^{-1} \dot{g}_{t}^{-1} \tilde{D}_{t}^{j+1} \cap \mathcal{A}_{\sigma_{t}}\right]\right) .
\end{aligned}
$$

We have used the fact that $D_{t}^{j+1} \subseteq P_{t}$. Recall for the last equation that $\mathcal{A}_{\sigma_{1}}=\emptyset$. Note that the number of pieces in the decomposition above is $j+\sum_{t=2}^{j}\left|\Lambda_{t}\right|$, so by the induction hypothesis

$$
\left|\Lambda_{j+1}\right|=j+\sum_{t=2}^{j}\left(2^{t-1}-1\right)=2^{j}-1 .
$$

Hence it is done for $j+1$. This completes the Step 2. Before proceeding to the rest of the proof, we draw the reader's attention to the next two remarks.

Remark 3.2. In step 2 we explained how the sets $\mathcal{A}_{\sigma_{j+1}}$ are obtained from the sets $P_{t}$ and $\mathcal{A}_{\sigma_{t}}$, where $1 \leq t<j+1$. In fact each $\mathcal{A}_{\sigma_{j+1}}$ is in one of the following two types:

$$
\begin{gathered}
\dot{g}_{t}^{-1} \tilde{D}_{t}^{j+1} \cap \tilde{P}_{t} \\
g_{\sigma_{t}}^{-1} \dot{g}_{t}^{-1} \tilde{D}_{t}^{j+1} \cap \mathcal{A}_{\sigma_{t}},
\end{gathered}
$$

for suitable $t<j+1$ and $\sigma_{t} \in \Lambda_{t}$. So, one can easily see that $\mathcal{A}_{\sigma_{j+1}} \cap \mathcal{A}_{\sigma_{t}} \neq \emptyset$ if and only if $\mathcal{A}_{\sigma_{j+1}} \subseteq \mathcal{A}_{\sigma_{t}}$. In this case for every $\sigma_{t}^{\prime} \neq \sigma_{t}, \mathcal{A}_{\sigma_{j+1}} \cap \mathcal{A}_{\sigma_{t}^{\prime}}=\emptyset$.

Remark 3.3. For $1 \leq r<t \leq p$ and $\sigma_{t} \in \Lambda_{t}$, either $\mathcal{A}_{\sigma_{t}}$ and $\tilde{P}_{r}$ are disjoint or $\mathcal{A}_{\sigma_{t}} \subseteq \tilde{P}_{r}$. To see this we apply Remark 3.2. Let $\mathcal{A}_{\sigma_{t}} \cap \tilde{P}_{r} \neq \emptyset$. If $\mathcal{A}_{\sigma_{t}}$ is of type (I), then $\mathcal{A}_{\sigma_{t}} \subseteq \tilde{P}_{k}$, for some $k<t$. In this case $\tilde{P}_{r} \cap \tilde{P}_{k} \neq \emptyset$, which is impossible unless $k=r$. Therefore $\mathcal{A}_{\sigma_{t}} \subseteq \tilde{P}_{r}$. Now suppose that $\mathcal{A}_{\sigma_{t}}$ is of type (II) and $l$ be the smallest natural number such that for some $\sigma_{l} \in \Lambda_{l}, \mathcal{A}_{\sigma_{t}} \subseteq \mathcal{A}_{\sigma_{l}}$ and $\mathcal{A}_{\sigma_{l}}$ is of 
type (II). Then $\mathcal{A}_{\sigma_{l}}=\tilde{P}_{m} \cap \dot{g}^{-1} \tilde{D}_{m}^{l} \subseteq \tilde{P}_{m}$, for some $m<l$, which is not possible unless $m=r$. This implies that $\mathcal{A}_{\sigma_{t}} \subseteq \tilde{P}_{r}$.

Step 3. We show that for $1 \leq t \leq p$ and $C \in Q_{t}$ there exists a family $\left\{\mathcal{M}_{\delta_{t}}^{C}, \quad \delta_{t} \in\right.$ $\left.\Gamma_{t}\right\}$ with cardinality $2^{t-1}$ of disjoint subsets of $\bigsqcup_{j=1}^{t} \tilde{P}_{j}$ and the members $\left\{h_{\delta_{t}}^{C}, \quad \delta_{t} \in\right.$ $\left.\Gamma_{t}\right\}$ of $G$ such that

$$
x_{0}(C)=\bigsqcup_{\delta_{t} \in \Gamma_{t}} h_{\delta_{t}}^{C} M_{\delta_{t}}^{C} .
$$

Since $Q_{t} \subseteq B_{t}$, by Step 1 we have

$$
\begin{aligned}
\tilde{Q}_{t} & \subseteq \tilde{B}_{t}=\dot{g}_{t} \tilde{A}_{t} \\
& =\dot{g}_{t} \tilde{P}_{t} \bigsqcup \dot{g}_{t}\left(\tilde{A}_{t} \backslash \tilde{P}_{t}\right) \\
& =\dot{g}_{t} \tilde{P}_{t} \bigsqcup \dot{g}_{t}\left(\bigsqcup_{\sigma_{t} \in \Lambda_{t}} g_{\sigma_{t}} \mathcal{A}_{\sigma_{t}}\right) \\
& =\dot{g}_{t} \tilde{P}_{t} \bigsqcup\left(\bigsqcup_{\sigma_{t} \in \Lambda_{t}} \dot{g}_{t} g_{\sigma_{t}} \mathcal{A}_{\sigma_{t}}\right)
\end{aligned}
$$

Clearly by Step 1 the number of pieces in this decomposition is $1+\left|\Lambda_{t}\right|=2^{t-1}$. Therefore for $C \in Q_{t}$, one has

$$
x_{0}(C)=\dot{g}_{t}\left(\tilde{P}_{t} \cap \dot{g}_{t}^{-1} x_{0}(C)\right) \bigsqcup\left(\bigsqcup_{\sigma_{t} \in \Lambda_{t}} \dot{g}_{t} g_{\sigma_{t}}\left(\mathcal{A}_{\sigma_{t}} \cap g_{\sigma_{t}}^{-1} \dot{g}_{t}^{-1} x_{0}(C)\right)\right) .
$$

Now set $M_{\delta_{0}}^{C}=\tilde{P}_{t} \cap \dot{g}_{t}^{-1} x_{0}(C)$ and $\left.M_{\delta_{t}}^{C}=\mathcal{A}_{\sigma_{t}} \cap g_{\sigma_{t}}^{-1} \dot{g}_{t}^{-1} x_{0}(C)\right)$, where $\sigma_{t} \in \Lambda_{t}$. Note that these sets are pairwise disjoint; $M_{\delta_{0}}^{C}$ is a subset of $\tilde{P}_{t}$ whereas the sets $M_{\delta_{t}}^{C}, \delta_{t} \in \Lambda_{t}$ are disjoint subsets of $\bigsqcup_{j=1}^{t-1} \tilde{P}_{j}$ and besides $\left(\bigsqcup_{j=1}^{t-1} \tilde{P}_{j}\right) \cap \tilde{P}_{t}=\emptyset$. Define $\Gamma_{t}=\left\{\delta_{0}\right\} \cup \Lambda_{t}, h_{\delta_{t}}^{C}=\dot{g}_{t} g_{\sigma_{t}}$, where $\delta_{t}=\sigma_{t} \in \Lambda_{t}$ and $h_{\delta_{t_{0}}}^{C}=\dot{g}_{t}$. Thus $\left\{M_{\delta_{t}}^{C}, \delta_{t} \in \Gamma_{t}\right\}$ is a family of pairwise disjoint subsets of $G$ and

$$
x_{0}(C)=\bigsqcup_{\delta_{t} \in \Gamma_{t}} h_{\delta_{t}}^{C} M_{\delta_{t}}^{C} .
$$

It is noticeable that for $1 \leq t \neq s \leq p, M_{\delta_{t}}^{C} \cap M_{\delta_{s}}^{C^{\prime}}=\emptyset$, where $C \in Q_{t}$ and $C^{\prime} \in Q_{s}$. We show this below. Without loss of generality assume that $t<s$. Letting $C \in Q_{t}$ and $C^{\prime} \in Q_{s}$, we need to prove that the following four equalities are satisfied

$$
\begin{aligned}
& M_{\delta_{0}}^{C} \cap M_{\delta_{0}}^{C^{\prime}}=\emptyset, \\
& M_{\delta_{t}}^{C} \cap M_{\delta_{0}}^{C^{\prime}}=\emptyset, \\
& M_{\delta_{0}}^{C} \cap M_{\delta_{s}}^{C^{\prime}}=\emptyset, \\
& M_{\delta_{t}}^{C} \cap M_{\delta_{s}}^{C^{\prime}}=\emptyset .
\end{aligned}
$$

But it is equivalent to show that

$$
\begin{aligned}
& {\left[\tilde{P}_{t} \cap \dot{g}_{t}^{-1} \tilde{Q}_{t}\right] \cap\left[\tilde{P}_{s} \cap \dot{g}_{s}^{-1} \tilde{Q}_{s}\right]=\emptyset} \\
& \left.\left[\tilde{P}_{s} \cap \dot{g}_{s}^{-1} \tilde{Q}_{s}\right] \cap\left[\mathcal{A}_{\sigma_{t}} \cap g_{\sigma_{t}}^{-1} \dot{g}_{t}^{-1} \tilde{Q}_{t}\right)\right]=\emptyset \\
& \left.\left[\tilde{P}_{t} \cap \dot{g}_{t}^{-1} \tilde{Q}_{t}\right] \cap\left[\mathcal{A}_{\sigma_{s}} \cap g_{\sigma_{s}}^{-1} \dot{g}_{s}^{-1} \tilde{Q}_{s}\right)\right]=\emptyset \\
& \left.\left.\left[\mathcal{A}_{\sigma_{t}} \cap g_{\sigma_{t}}^{-1} \dot{g}_{t}^{-1} \tilde{Q}_{t}\right)\right] \cap\left[\mathcal{A}_{\sigma_{s}} \cap g_{\sigma_{s}}^{-1} \dot{g}_{s}^{-1} \tilde{Q}_{s}\right)\right]=\emptyset
\end{aligned}
$$


The first two equalities are trivial, since for each $r \neq s, P_{r} \cap P_{s}=\emptyset$ and besides, $\mathcal{A}_{\sigma_{t}} \subseteq \bigsqcup_{r=1}^{t-1} \tilde{P}_{r}$ and $t<s$. We now prove (20). If $\mathcal{A}_{\sigma_{s}}$ is of type (I), then there exists $l<s$ such that $\mathcal{A}_{s}=\dot{g}_{l}^{-1} \tilde{D}_{l}^{s} \cap \tilde{P}_{l}$. So

$$
\left.\left[\tilde{P}_{t} \cap \dot{g}_{t}^{-1} \tilde{Q}_{t}\right] \cap\left[\mathcal{A}_{\sigma_{s}} \cap g_{\sigma_{s}}^{-1} \dot{g}_{s}^{-1} \tilde{Q}_{s}\right)\right] \subseteq \tilde{P}_{l} \cap \tilde{P}_{t}
$$

The set $\tilde{P}_{l} \cap \tilde{P}_{t}$ is clearly empty if $l \neq t$. If $l=t$,

$$
\left[\tilde{P}_{t} \cap \dot{g}_{t}^{-1} \tilde{Q}_{t}\right] \cap\left[\mathcal{A}_{\sigma_{s}} \cap g_{\sigma_{s}}^{-1} \dot{g}_{s}^{-1} \tilde{Q}_{s}\right] \subseteq \tilde{P}_{l} \cap \dot{g}_{l}^{-1} \tilde{Q}_{l} \cap \dot{g}_{l}^{-1} \tilde{D}_{l}^{s}
$$

and $\tilde{P}_{l} \cap \dot{g}_{l}^{-1} \tilde{Q}_{l} \cap \dot{g}_{l}^{-1} \tilde{D}_{l}^{s}$ is also empty since $\tilde{Q}_{l} \cap \tilde{D}_{l}^{s}$, by (15). Now let $\mathcal{A}_{\sigma_{s}}$ be of type (II) and $k$ be the smallest natural number such that $\mathcal{A}_{\sigma_{s}} \subseteq \mathcal{A}_{\sigma_{k}}$ and $\mathcal{A}_{\sigma_{k}}$ is of type (II). This implies that $\mathcal{A}_{\sigma_{k}} \subseteq \tilde{P}_{l} \cap \dot{g}_{l}^{-1} \tilde{D}_{l}^{k}$, for some $l<k$. If (20) does not satisfy, then by Remark 3.3. $\mathcal{A}_{\sigma_{s}} \subseteq \tilde{P}_{t}$ and this is impossible unless $t=l$. So $\mathcal{A}_{\sigma_{s}} \subseteq \mathcal{A}_{\sigma_{k}} \subseteq \dot{g}_{t}^{-1} \tilde{D}_{t}^{k} \cap \tilde{P}_{t}$. Taking into account that $D_{t}^{k} \cap \tilde{Q}_{t}=\emptyset$, we have

$$
\left[\tilde{P}_{t} \cap \dot{g}_{t}^{-1} \tilde{Q}_{t}\right] \cap \mathcal{A}_{\sigma_{s}} \subseteq \dot{g}_{t}^{-1} \tilde{Q}_{t} \cap \dot{g}_{t}^{-1} \tilde{D}_{t}^{k}=\emptyset .
$$

This contradicts our assumption.

To prove (21) let $\mathcal{A}_{\sigma_{s}} \cap \mathcal{A}_{\sigma_{t}} \neq \emptyset$. By Remark $3.2 \mathcal{A}_{\sigma_{s}} \subseteq \mathcal{A}_{\sigma_{t}}$. In fact $\mathcal{A}_{\sigma_{s}}=$ $\mathcal{A}_{\sigma_{t}} \cap g_{\sigma_{t}}^{-1} \dot{g}_{t}^{-1} \tilde{D}_{t}^{s}$. Thus we have

$$
\left[\mathcal{A}_{\sigma_{t}} \cap g_{\sigma_{t}}^{-1} \dot{g}_{t}^{-1} \tilde{Q}_{t}\right] \cap\left[\mathcal{A}_{\sigma_{s}} \cap g_{\sigma_{s}}^{-1} \dot{g}_{s}^{-1} \tilde{Q}_{s}\right] \subseteq \mathcal{A}_{\sigma_{t}} \cap g_{\sigma_{t}}^{-1} \dot{g}_{t}^{-1} \tilde{Q}_{t} \cap g_{\sigma_{t}}^{-1} \dot{g}_{t}^{-1} \tilde{D}_{t}^{s}=\emptyset
$$

because $\tilde{Q}_{t} \cap \tilde{D}_{t}^{s}=\emptyset$.

Step 4. $P_{1}, P_{2}, \ldots, P_{p}$ are pairwise disjoint and for each $C \in \operatorname{Con}(\mathfrak{g}, \mathcal{E})$

$$
\sum_{t=1}^{p} V_{t}^{\prime}(C) \in\{0,1\}
$$

For $C \in \operatorname{Con}(\mathfrak{g}, \mathcal{E})$ we put $z_{C}=\alpha_{C}+\sum_{t=1}^{p} V_{t}^{\prime}(C)$. This way, if $\sum_{t=1}^{p} V_{t}^{\prime}(C)=1$, then $z_{c} \geq 2$. So, by (13) there are at least two numbers $1 \leq t_{1}^{C}, t_{2}^{C} \leq p$ such that $C \in Q_{t_{1}^{C}}$ and $C \in Q_{t_{2}^{C}}$. Therefore by Step 2 ,

$$
x_{0}(C)=\bigsqcup_{\delta_{t_{1}} \in \Gamma_{t_{1}^{C}}} h_{\delta_{1}^{C}}^{C} M_{\delta_{t_{1}^{C}}}^{C}=\bigsqcup_{\delta_{t_{2}^{C}} \in \Gamma_{t_{2}^{C}}} h_{\delta_{t_{2}^{C}}^{C}}^{C} M_{\delta_{t_{2}^{C}}^{C}}^{C} .
$$

On the other hand, if $\sum_{t=1}^{p} V_{t}^{\prime}(C)=0$, then $z_{c} \geq 1$, so by (13) there is at least one number $1 \leq t_{C} \leq p$ such that $C \in Q_{t^{C}}$. Therefore by Step 2,

$$
x_{0}(C)=\bigsqcup_{\delta_{t} C \in \Gamma_{t} C} h_{\delta_{t} C}^{C} M_{\delta_{t} C}^{C}
$$

Thus

$$
\begin{gathered}
G \supseteq\left[\bigsqcup_{\sum V_{i}^{\prime}(C)=1}\left(\bigsqcup_{t_{1}^{C} \in \Gamma_{t_{1}^{C}}} \mathcal{M}_{\delta_{t_{1}}}^{C}\right) \bigsqcup\left(\bigsqcup_{\delta_{2}^{C} \in \Gamma_{t_{2}^{C}}} \mathcal{M}_{\delta_{t_{2}^{C}}^{C}}^{C}\right)\right] \\
\bigsqcup\left[\bigsqcup_{\sum V_{i}^{\prime}(C)=0} \bigsqcup_{\delta_{t} C \Gamma_{t^{C}}} \mathcal{M}_{\delta_{t^{C}}}^{C}\right] \bigsqcup\left[\bigsqcup_{\sum V_{i}^{\prime}(C)=0} x_{0}(C)\right]
\end{gathered}
$$


Observe that the sets in the right hand side of above inclusion are pairwise disjoint because

$$
\left\{C \in \operatorname{Con}(\mathfrak{g}, \mathcal{E}), \quad \sum V_{i}^{\prime}(C)=0\right\} \subseteq \operatorname{Con}(\mathfrak{g}, \mathcal{E}) \backslash \bigsqcup_{i=1}^{p} P_{i}
$$

i.e.,

$$
\left(\bigsqcup_{\sum V_{i}^{\prime}(C)=0} x_{0}(C)\right) \bigcap\left(\bigsqcup_{i=1}^{p} \tilde{P}_{i}\right)=\emptyset
$$

and the sets $M_{\delta_{t}}^{C}, \delta_{t} \in \Gamma_{t}$ are pairwise disjoint subsets of $\bigsqcup_{i=1}^{p} \tilde{P}_{i}$ (take into account that $t_{1}^{C}$, and $t_{2}^{C}$ are distinct numbers). Based on our choice of sets

$$
\begin{aligned}
& G=\left[\bigsqcup_{\sum V_{i}^{\prime}(C)=1}\left(\bigsqcup_{\delta_{t_{1}^{C}} \in \Gamma_{t^{C}}} h_{\delta_{t_{1}^{C}}^{C}}^{C} M_{\delta_{1}}^{C}\right)\right] \bigsqcup\left[\bigsqcup_{\sum V_{i}^{\prime}(C)=0}\left(\bigsqcup_{\delta_{t} C} \bigsqcup_{t^{C}} h_{\delta_{t} C}^{C} M_{\delta_{t} C}^{C}\right)\right] \\
& G=\left[\bigsqcup_{\sum V_{i}^{\prime}(C)=1}\left(\bigsqcup_{t_{2}^{C}}^{(} \bigsqcup_{t_{2}^{C}} h_{\delta_{t_{2}^{C}}^{C}}^{C} M_{\delta_{t_{2}^{C}}^{C}}^{C}\right)\right] \bigsqcup\left[\bigsqcup_{\sum V_{i}^{\prime}(C)=0} x_{0}(C)\right] \text {, }
\end{aligned}
$$

which is a paradoxical decomposition of $G$ after omitting the empty sets from this decomposition. This decomposition is complete if for each $C \in C o n(\mathfrak{g}, \mathcal{E})$,

$$
\sum_{i=1}^{n} V_{i}^{\prime}(C)=a_{C}=1
$$

Otherwise the decomposition is not complete but by a process described in the proof of [7, Proposition 1.2] it can be changed into a complete one.

Corollary 3.4. Using the notations of the proof of Theorem 3.1, if for every $C \in$ $\operatorname{Con}(\mathfrak{g}, \mathcal{E}), a_{C}=1$, then $\tau(G) \leq(\ell-1)\left(2^{p}-1\right)$.

Proof. By the definition of configuration the cardinality of each $B_{t}$ cannot be more than $\ell-1$, where $|\operatorname{Con}(\mathfrak{g}, \mathcal{E})|=\ell$. The Step 3 in the proof of Theorem 3.1 and the explanation following Proposition 2.6 yields

$$
\begin{aligned}
\tau(G) & \leq \sum_{t=1}^{p}\left|\Lambda_{t}\right|\left|Q_{t}\right| \\
& \leq \sum_{t=1}^{p} 2^{t-1}\left|B_{t}\right| \\
& \leq \sum_{t=1}^{p} 2^{t-1}(\ell-1) \\
& \leq(\ell-1)\left(2^{p}-1\right) .
\end{aligned}
$$

Remark 3.5. In [7] the authors constructed the paradoxical decomposition under the paradoxical condition. That is, a subsystem of $E q(\mathfrak{g}, \mathcal{E})$ is equivalent to $B X=0$, 
where each row of $B$ has nonnegative entries and is of the form $\sum_{i=1}^{m}\left(L_{i}^{j_{i}}-L_{i}^{k_{i}}\right)$, for some $j_{i}, k_{i} \in\{0, \ldots, n\}$, where $L_{i}^{j}$ is the coefficient vector of the equation

$$
\sum_{x_{j}(C) \subseteq E_{i}} f_{C}-\sum_{x_{0}(C) \subseteq E_{i}} f_{C}=0
$$

and in addition $B$ has no zero column. Let $R^{s}=\sum_{i=1}^{m}\left(L_{i}^{j_{i}^{s}}-L_{i}^{k_{i}^{s}}\right)$ be s-th row of B. Then

so

$$
\sum_{i=1}^{m}\left(\sum_{x_{j_{i}^{s}}(C) \subseteq E_{i}} f_{C}-\sum_{x_{k_{i}^{s}}(C) \subseteq E_{i}} f_{C}\right)=\sum_{C} R^{s}(C) f_{C},
$$

$$
\sum_{i=1}^{m}\left(\sum_{x_{j_{i}^{s}}(C) \subseteq E_{i}} f_{C}\right)=\sum_{i=1}^{m}\left(\sum_{x_{k_{i}^{s}}(C) \subseteq E_{i}} f_{C}\right)+\sum_{C} R^{s}(C) f_{C}
$$

therefore we have

$$
\sum_{i=1}^{m}\left(\sum_{x_{0}(C) \subseteq E_{i}} f_{C}\right)=\sum_{i=1}^{m}\left(\sum_{x_{0}(C) \subseteq E_{i}} f_{C}\right)+\sum_{C} R^{s}(C) f_{C} .
$$

But $\operatorname{Con}(\mathfrak{g}, \mathcal{E})=\bigsqcup_{i=1}^{m} \bigsqcup_{x_{0}(C) \subseteq E_{i}} x_{0}(C)$. Consequently we can replace $B$ by $W-V$, where

$$
W=\left(\left(\begin{array}{c}
1,1, \ldots, 1 \\
1,1, \ldots, 1 \\
\vdots \\
1,1, \ldots, 1
\end{array}\right)+\left(\begin{array}{c}
R_{1} \\
R_{2} \\
\vdots \\
R_{p}
\end{array}\right)\right) \text { and } V=\left(\begin{array}{c}
1,1, \ldots, 1 \\
1,1, \ldots, 1 \\
\vdots \\
1,1, \ldots, 1
\end{array}\right) .
$$

Since by assumption each $R_{s}$ has nonnegative entries, $W_{s}-V_{s}-V_{s+1}$ has entries $\geq-1$. Finally $B$ has no zero column, so if $\sum_{s}\left(W_{s}-V_{s}\right)=\left(\alpha_{C}\right)_{C}$, then $\alpha_{C}>0$. This shows that the new system satisfies the normality condition in the statement of Theorem 3.1.

\section{Graph interpretation}

In the current section we assign a graph to a group which helps us construct the paradoxical decompositions and compute the Tarski numbers.

Definition 4.1. Let $G$ be a group and $\mathfrak{g}=\left(g_{1}, \ldots, g_{n}\right)$ and $\mathcal{E}=\left\{E_{1}, \ldots, E_{m}\right\}$ be a finite string of elements of $G$ and a finite partition for $G$, respectively. $\Gamma=\Gamma(G, \mathfrak{g}, \mathcal{E})$ is a graph constructed as follows

- The vertex set of $\Gamma$ is identified with $\ell$-tuples of nonnegative integers, where $|\operatorname{Con}(\mathfrak{g}, \mathcal{E})|=\ell$ :

$$
V(\Gamma):=\left\{\left(a_{C}\right)_{C \in \operatorname{Con}(\mathfrak{g}, \mathcal{E})} ; \quad a_{C} \in \mathbb{N} \cup\{0\}\right\}
$$

- There exists a directed edge from the vertex $A=\left(a_{C}\right)$ to $B=\left(b_{C}\right)$ only if $a_{C} \in\{0,1\}$ and there are disjoint subfamilies

$$
\mathcal{A}_{1}^{C}, \ldots, \mathcal{A}_{b_{C}}^{C} \quad(C \in \operatorname{Con}(\mathfrak{g}, \mathcal{E}))
$$


of a partition of $\bigsqcup_{a_{C} \neq 0} x_{0}(C)$ such that for all $C \in \operatorname{Con}(\mathfrak{g}, \mathcal{E})$

$$
x_{0}(C)=\bigsqcup_{A \in \mathcal{A}_{j}^{C}} g_{A} A, \quad 1 \leq j \leq b_{C},
$$

for suitable subsets $\left\{g_{A}, A \in \mathcal{A}_{j}^{C}\right\}, 1 \leq j \leq b_{C}$, of $G$.

Proposition 4.2. If $\Gamma$ contains adjacent vertices $A=\left(a_{C}\right)_{C \in C o n(\mathfrak{g}, \mathcal{E})}$ and $B=$ $\left(b_{C}\right)_{C \in \operatorname{Con}(\mathfrak{g}, \mathcal{E})}$ with $a_{C} \in\{0,1\}$ and $\alpha_{C}:=b_{C}-a_{C}>0$, then $G$ admits a paradoxical decomposition in terms of $\mathfrak{g}$ and $\mathcal{E}$.

Proof. By the assumption for each $C, b_{C} \geq a_{C}+1$. In other words

$$
b_{C} \geq \begin{cases}1, & \text { if } a_{C}=0 \\ 2, & \text { if } a_{C} \neq 0 .\end{cases}
$$

Hence by Definition 4.1 there exists a partition $\mathcal{P}$ of $\bigsqcup_{a_{C} \neq 0} x_{0}(C)$ with the following properties:

- If $a_{C} \neq 0$, there are $\mathcal{A}_{1}^{C}, \mathcal{A}_{2}^{C} \subseteq \mathcal{P}$ and $\left\{g_{A}, A \in \mathcal{A}_{1}^{C}\right\},\left\{g_{B}, B \in \mathcal{A}_{2}^{C}\right\} \subseteq G$ such that

$$
x_{0}(C)=\bigsqcup_{A \in \mathcal{A}_{1}^{C}} g_{A} \cdot A=\bigsqcup_{B \in \mathcal{A}_{2}^{C}} g_{B} \cdot B .
$$

- If $a_{C}=0$, there are $\mathcal{A}^{C} \subseteq \mathcal{P}$ and $\left\{g_{D}, D \in \mathcal{A}^{C}\right\} \subseteq G$ such that

$$
x_{0}(C)=\bigsqcup_{D \in \mathcal{A}^{C}} g_{D} \cdot D \text {. }
$$

- All the above subfamilies of $\mathcal{P}$ are pairwise disjoint.

For convenience denote the set $\bigsqcup_{a_{C}=0} x_{0}(C)$ by $E$. We know that $G=\bigsqcup_{C \in C o n(\mathfrak{g}, \mathcal{E})} x_{0}(C)$. Then we have

$$
G=E \bigsqcup\left(\bigsqcup_{a_{C} \neq 0} \bigsqcup_{A \in \mathcal{A}_{1}^{C}} g_{A} \cdot A\right)
$$

and

$$
G=\left(\bigsqcup_{a_{C}=0} \bigsqcup_{D \in \mathcal{A}^{C}} g_{D} \cdot D\right) \bigsqcup\left(\bigsqcup_{a_{C} \neq 0} \bigsqcup_{B \in \mathcal{A}_{2}^{C}} g_{B} \cdot B\right)
$$

which give a (not necessarily complete) paradoxical decomposition for $G$. To make it complete, one can apply the proof of [7, Proposition 1.2] as usual.

Theorem 4.3. Let $G$ be a group and $\mathfrak{g}=\left(g_{1}, \ldots, g_{n}\right)$ and $\mathcal{E}=\left\{E_{1}, \ldots, E_{m}\right\}$ be a finite string of elements of $G$ and a finite partition for $G$, respectively. If $E q(\mathfrak{g}, \mathcal{E})$ has no nonnegative nonzero solution with a normal subsystem, then $\Gamma=\Gamma(G, \mathfrak{g}, \mathcal{E})$ includes vertices $A=\left(a_{C}\right)_{C \in C o n(\mathfrak{g}, \mathcal{E})}$ and $B=\left(b_{C}\right)_{C \in C o n(\mathfrak{g}, \mathcal{E})}$ with $a_{C} \in\{0,1\}$ and $\alpha_{C}:=b_{C}-a_{C}>0$.

Proof. Using the notations of the proof of Theorem 3.1, $A=\sum V_{i}^{\prime}$ and $B=\sum W_{i}^{\prime}$, are desired vertices because first, $B-A>0$ and $\sum V^{\prime}(C) \in\{0,1\}$, and second, by (17) they satisfy the second condition of the definition of $\Gamma(G, \mathfrak{g}, \mathcal{E})$. 
4.1. Diagrams associated with configuration equations. Here we present a kind of diagram associated with configuration equations. It could be of great importance in order to reduce the complexity of the proofs in the previous section.

- Let $A, B_{1}, \ldots, B_{t}$ be subsets of $\operatorname{Con}(\mathfrak{g}, \mathcal{E})$. If there is $g \in G$ and there is a partition $\left\{A_{1}, \ldots, A_{t}\right\}$ for $\tilde{A}=\bigsqcup_{C \in A} x_{0}(C)$ such that

$$
\tilde{B}_{j}=g A_{j}, \quad 1 \leq j \leq t,
$$

we use the following tree diagram

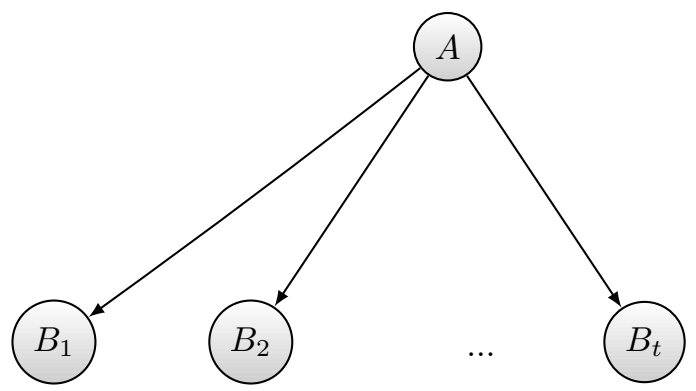

Clearly if $D \subseteq A$, and $E_{j}=\tilde{B}_{j} \cap g \tilde{D}$, we also have a similar diagram with directed paths from $D$ to $E_{1}, \ldots, E_{t-1}, E_{t}$.

- By the diagram

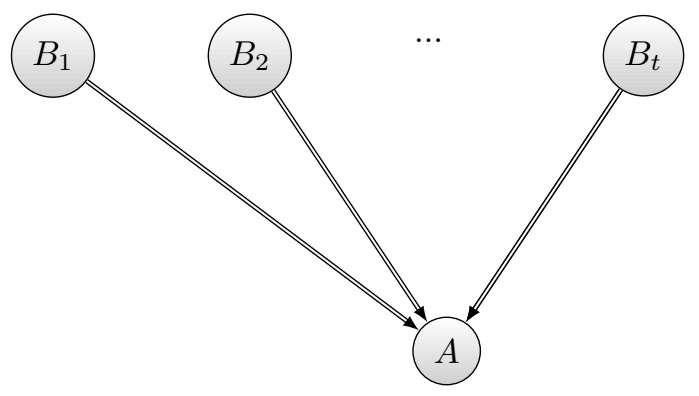

we mean $A=\bigsqcup_{i=1}^{t} B_{i}$.

- Let $A, B \subseteq \operatorname{Con}(\mathfrak{g}, \mathcal{E})$. In the sequel we denote a directed path of any kind of above two types from $A$ to $B$ by $[A, B]$. Inductively $\left[A, B_{1}, B_{2}, \ldots, B_{n}\right]$ is a path which connects the sets $A, B_{1}, B_{2}, \ldots, B_{n}$ through the sequence of $n$ single paths $\left[A, B_{1}\right],\left[B_{1}, B_{2}\right], \ldots,\left[B_{n-1}, B_{n}\right]$.

In the proof of Theorem 3.1 according to the normality condition we have the possibility to borrow the configurations in $A_{t} \backslash P_{t}$ from $B_{1}, \ldots, B_{t-1}$ so that the (not necessarily disjoint) subsets $A_{1}, \ldots, A_{p}$ turn into disjoint subsets $P_{1}, \ldots, P_{p}$ and it is enough for us to construct a paradoxical decomposition of $G$ and to get an upper bound for $\tau(G)$. We Apply the above diagrams to make the proof of that theorem more clear to the reader. Using the same notations, by (15) we have $\tilde{P}_{1}=\tilde{A}_{1}=\dot{g}_{1}^{-1} \tilde{B}_{1}=\dot{g}_{1}^{-1} \tilde{Q}_{1} \sqcup \dot{g}_{1}^{-1} \tilde{D}_{1}^{2} \sqcup \cdots \sqcup \dot{g}_{1}^{-1} \tilde{D}_{1}^{p}$. So, there are $p$ directed paths from $P_{1}=A_{1}$ to $D_{1}^{2}, \ldots, D_{1}^{p}$ and $Q_{1}$. Also $\tilde{D}_{1}^{2} \sqcup \tilde{P}_{2}=\tilde{A}_{2}=\dot{g}_{2}^{-1} \tilde{B}_{2}=$ $\dot{g}_{2}^{-1} \tilde{Q}_{2} \bigsqcup \dot{g}_{2}^{-1} \tilde{D}_{2}^{3} \sqcup \cdots \sqcup \dot{g}_{2}^{-1} \tilde{D}_{2}^{p}$. So, there are $p-1$ directed paths from $D_{1}^{2}$ to $D_{2}^{3}, \ldots, D_{2}^{p}$ and $Q_{2}$ and the same number of paths from $P_{2}$ to $D_{2}^{3}, \ldots, D_{2}^{p}$ and $Q_{2}$. 

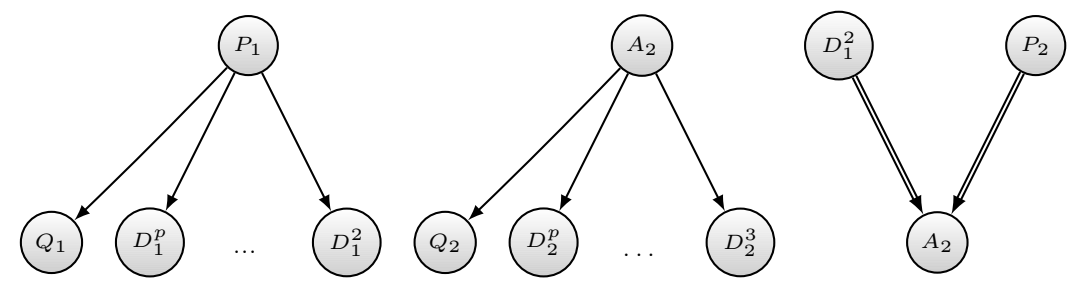

Gluing these diagrams we obtain two paths from $D_{1}^{2}$ and $P_{2}$ to $Q_{2}$ and so on.

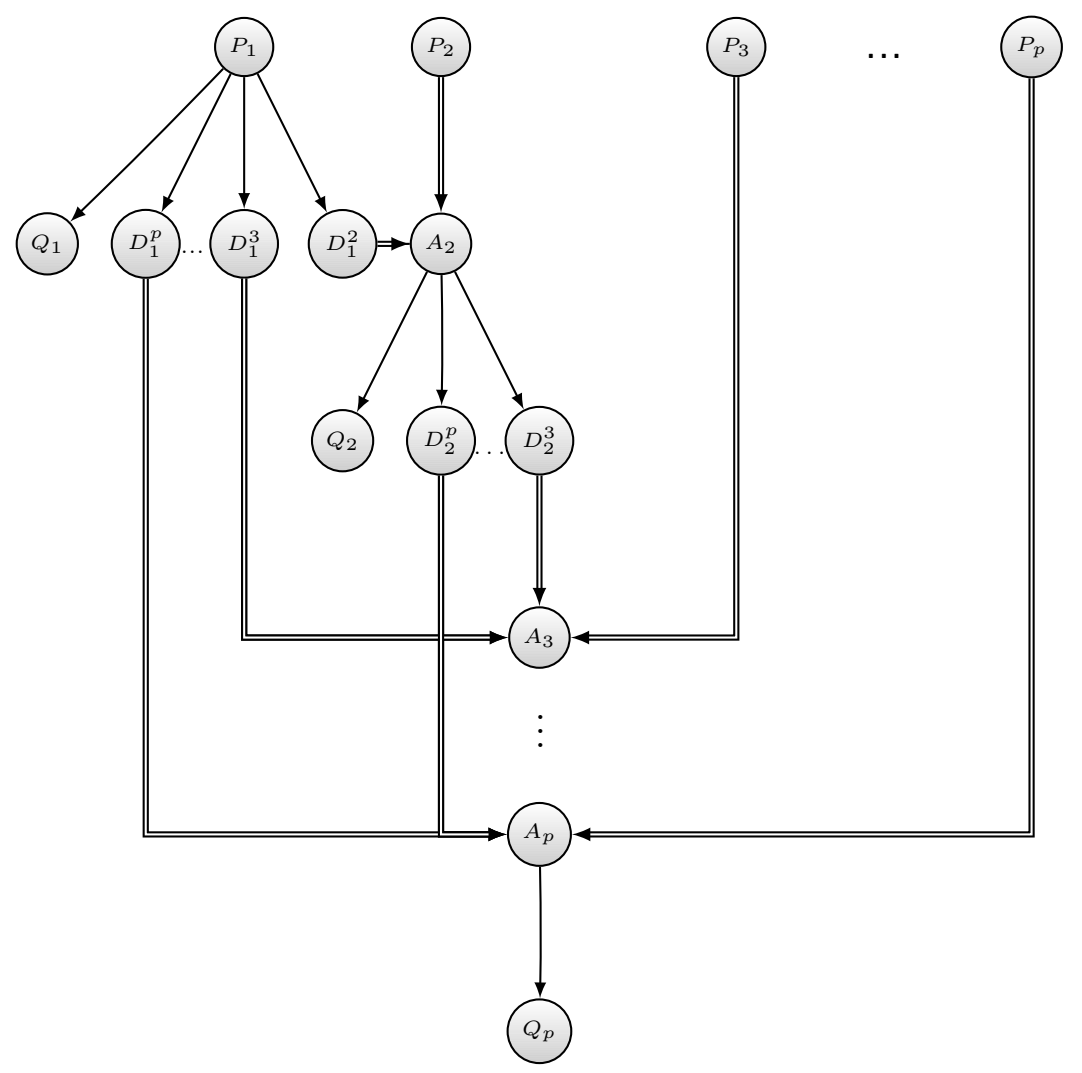


Our diagram contains the following $2^{p}-1$ paths

$$
\begin{aligned}
& {\left[P_{1}, Q_{1}\right],} \\
& {\left[P_{1}, D_{1}^{2}, A_{2}, Q_{2}\right],\left[P_{2}, A_{2}, Q_{2}\right],} \\
& {\left[P_{1}, D_{1}^{3}, A_{3}, Q_{3}\right],\left[P_{1}, D_{1}^{2}, A_{2}, D_{2}^{3}, A_{3}, Q_{3}\right],\left[P_{2}, A_{2}, D_{2}^{3}, A_{3}, Q_{3}\right],\left[P_{3}, A_{3}, Q_{3}\right],} \\
& \vdots \\
& {\left[P_{1}, D_{1}^{p}, A_{p}, Q_{p}\right], \ldots,\left[P_{1}, D_{1}^{2}, A_{2}, D_{2}^{p}, A_{p}, Q_{p}\right], \ldots,\left[P_{p}, A_{p}, Q_{p}\right] .}
\end{aligned}
$$

Depending on the members of $Q_{1}, \ldots, Q_{p}$, these paths can be continued one more step. In fact $\bigsqcup_{t=1}^{p} P_{t}$ admits a partition with at most $2^{p}-1$ pieces such that with suitable coefficients construct the sets $\bigsqcup_{C \in Q_{t}} x_{0}(C), 1 \leq t \leq p$, separately (see proof of Theorem 3.1). $2^{t-1}$ pieces in the partition are used to make $\tilde{Q}_{t}$. These pieces are divided into $2^{t-1}\left|Q_{t}\right|$ new pieces to construct all the sets $x_{0}(C), C \in Q_{t}$. To have a paradoxical decomposition each $x_{0}(C), C \in C o n(\mathfrak{g}, \mathcal{E})$ must be obtained at least two times. If $\sum_{t=1}^{p} V_{t}^{\prime}(C)=1$, then $\sum_{t=1}^{p} W_{t}^{\prime}(C) \geq 2$. In other words, $C$ belongs to at least two $Q_{t} s$ and it is done. Otherwise, $C$ may appear in only one $Q_{t}$ and we have to add the single set $\left\{C, \sum_{t=1}^{p} V_{t}^{\prime}(C)=0 \tilde{\}}\right.$ to the pieces of our paradoxical decomposition. Therefore $\tau(G) \leq \sum_{t=1}^{p} 2^{t-1}\left|Q_{t}\right|+\mathbf{1}$. It is to be noted that $\sum_{t=1}^{p} 2^{t-1}\left|Q_{t}\right|$ is the maximum possible number of mentioned paths.

Naturally to make this observation more accurate, we have to choose a procedure in which the number of pieces in the paradoxical decomposition is as small as possible. This process depends directly on the set of configurations given in the statement of problem. We explain it in the next subsection.

4.2. Examples. We end the paper with some examples. Our examples will be based on the sets of configurations instead of the elements of the group. As the paradoxical decomposition of a group is not unique, different diagrams also exist. One can find a more precise Tarski number's upper bound by counting the paths from the top to the bottom in a minimal diagram (a diagram with minimum number of paths). It is noticeable that a minimal diagram is not necessarily a connected one.

Example 4.4. Let $C_{1}=(1,2,3,2), C_{2}=(1,3,1,3), C_{3}=(2,1,2,2), C_{4}=$ $(3,3,1,2)$ and $C_{5}=(3,3,2,1)$ be configurations corresponding to the configuration 
pair $(\mathfrak{g}, \mathcal{E})$ of a group $G$. Consider the following equations

$$
\begin{aligned}
\sum_{x_{1}(C) \subseteq E_{1}} f_{C} & =\sum_{x_{0}(C) \subseteq E_{1}} f_{C}, \\
\sum_{x_{1}(C) \subseteq E_{1}} f_{C} & =\sum_{x_{0}(C) \subseteq E_{1}} f_{C} \\
\sum_{x_{3}(C) \subseteq E_{1}} f_{C} & =\sum_{x_{0}(C) \subseteq E_{1}} f_{C}, \\
\sum_{x_{3}(C) \subseteq E_{1}} f_{C} & =\sum_{x_{0}(C) \subseteq E_{1}} f_{C} \\
\sum_{x_{0}(C) \subseteq E_{2}} f_{C} & =\sum_{x_{2}(C) \subseteq E_{2}} f_{C}, \\
\sum_{x_{1}(C) \subseteq E_{2}} f_{C} & =\sum_{x_{2}(C) \subseteq E_{2}} f_{C} \\
\sum_{x_{3}(C) \subseteq E_{3}} f_{C} & =\sum_{x_{0}(C) \subseteq E_{3}} f_{C}
\end{aligned}
$$

which are the next equations, respectively

$$
\begin{aligned}
& f_{C_{3}}=f_{C_{1}}+f_{C_{2}}, \\
& f_{C_{3}}=f_{C_{1}}+f_{C_{2}}, \\
& f_{C_{5}}=f_{C_{3}}, \\
& f_{C_{5}}=f_{C_{3}}, \\
& f_{C_{3}}=f_{C_{3}}+f_{C_{5}}, \\
& f_{C_{1}}=f_{C_{3}}+f_{C_{5}}, \\
& f_{C_{2}}=f_{C_{4}}+f_{C_{5}} .
\end{aligned}
$$

Then $A=\left(\begin{array}{lllll}0 & 0 & 1 & 0 & 0 \\ 0 & 0 & 1 & 0 & 0 \\ 0 & 0 & 0 & 0 & 1 \\ 0 & 0 & 0 & 0 & 1 \\ 0 & 0 & 1 & 0 & 0 \\ 1 & 0 & 0 & 0 & 0 \\ 0 & 1 & 0 & 0 & 0\end{array}\right)$ and $B=\left(\begin{array}{lllll}1 & 1 & 0 & 0 & 0 \\ 1 & 1 & 0 & 0 & 0 \\ 0 & 0 & 1 & 0 & 0 \\ 0 & 0 & 1 & 0 & 0 \\ 0 & 0 & 1 & 0 & 1 \\ 0 & 0 & 1 & 0 & 1 \\ 0 & 0 & 0 & 1 & 1\end{array}\right)$ are the corresponding coefficient matrices. Setting $\pi=(27)(46)$, one has

$$
T P_{\pi}(B-A)-P_{\pi}^{+} A=\left(\begin{array}{ccccc}
1 & 0 & -1 & 0 & 0 \\
1 & 0 & -1 & 1 & 0 \\
0 & 0 & 0 & 1 & 0 \\
0 & 0 & 0 & 1 & 1 \\
0 & 0 & 1 & 1 & 1 \\
0 & 0 & 1 & 1 & 1 \\
1 & 1 & 0 & 1 & 1
\end{array}\right)
$$


Therefore this system is normal. The equations corresponding to $T P_{\pi}(B-A)$ are

$$
\begin{aligned}
& f_{C_{3}}=f_{C_{1}}+f_{C_{2}}, \\
& f_{C_{2}}=f_{C_{4}}+f_{C_{5}}, \\
& f_{C_{5}}=f_{C_{3}}, \\
& f_{C_{1}}=f_{C_{3}}+f_{C_{5}}, \\
& f_{C_{3}}=f_{C_{3}}+f_{C_{5}}, \\
& f_{C_{5}}=f_{C_{3}}, \\
& f_{C_{3}}=f_{C_{1}}+f_{C_{2}} .
\end{aligned}
$$

We use the notations of the proof of Theorem 3.1. In this example $p=7, A_{1}=$ $\left\{C_{3}\right\}, A_{2}=\left\{C_{2}\right\}, A_{3}=\left\{C_{5}\right\}, A_{4}=\left\{C_{1}\right\}, A_{5}=\left\{C_{3}\right\}, A_{6}=\left\{C_{5}\right\}, A_{7}=\left\{C_{3}\right\}$, $B_{1}=\left\{C_{1}, C_{2}\right\}, B_{2}=\left\{C_{4}, C_{5}\right\}, B_{3}=\left\{C_{3}\right\}, B_{4}=\left\{C_{3}, C_{5}\right\}, B_{5}=\left\{C_{3}, C_{5}\right\}, B_{6}=$ $\left\{C_{3}\right\}, B_{7}=\left\{C_{1}, C_{2}\right\}$. The initial diagram is
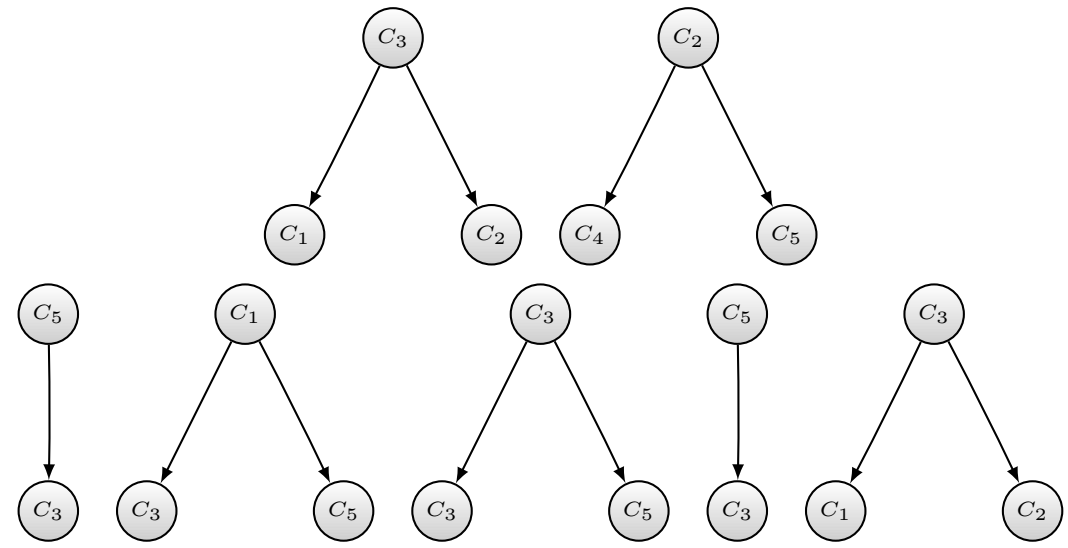

Applying the process in the proof of Theorem, we have $P_{1}=\left\{C_{3}\right\}, P_{2}=\left\{C_{2}\right\}, P_{3}=$ $\left\{C_{5}\right\}, P_{4}=\left\{C_{1}\right\}, P_{5}=\emptyset, P_{6}=\emptyset, P_{7}=\emptyset, Q_{1}=\left\{C_{1}, C_{2}\right\}, Q_{2}=\left\{C_{4}\right\}, Q_{3}=\emptyset, Q_{4}=$ $\left\{C_{3}, C_{5}\right\}, Q_{5}=\left\{C_{5}\right\}, Q_{6}=\left\{C_{3}\right\}, Q_{7}=\left\{C_{1}, C_{2}\right\}$. The associated diagram is

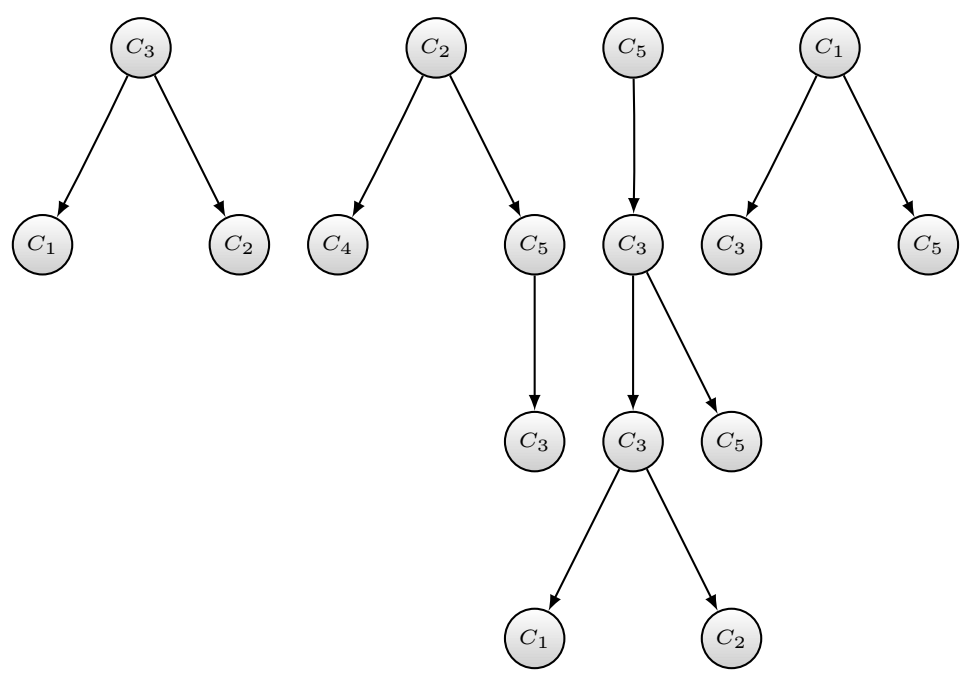


The condition of normality enables us to have disjoint subsets $P_{1}, \ldots, P_{p}$ which produce $\operatorname{Con}(\mathfrak{g}, \mathcal{E})$ two times (see proof of Theorem 3.1). By Corollary 3.4. $\tau(G) \leq$ $(\ell-1)\left(2^{p}-1\right)=508(\ell=5$ and $p=7)$. This is not a very accurate upper bound. Changing the diagram to a minimal one, helps us compute the most precise bound $\tau(G)$. Look at the following diagram

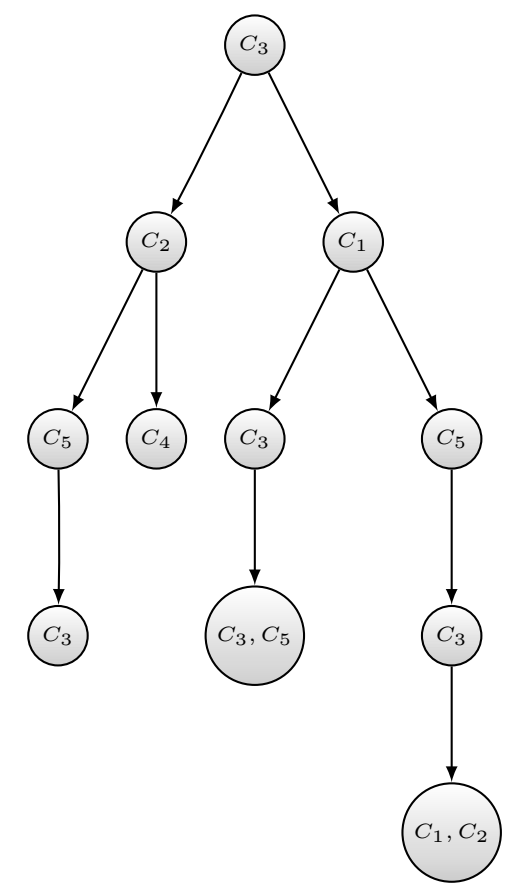

According to (5) we have $g_{1} x_{0}\left(C_{3}\right)=x_{0}\left(C_{1}\right) \bigsqcup x_{0}\left(C_{2}\right), g_{3} x_{0}\left(C_{2}\right)=x_{0}\left(C_{4}\right) \bigsqcup x_{0}\left(C_{5}\right)$, $g_{2}^{-1} g_{1} x_{0}\left(C_{1}\right)=x_{0}\left(C_{3}\right) \bigsqcup x_{0}\left(C_{5}\right), g_{2}^{-1} x_{0}\left(C_{3}\right)=x_{0}\left(C_{3}\right) \bigsqcup x_{0}\left(C_{5}\right)$ and $g_{3} x_{0}\left(C_{5}\right)=$ $x_{0}\left(C_{3}\right)$.

Then $x_{0}\left(C_{3}\right)=\Delta_{1} \bigsqcup \Delta_{2} \bigsqcup \Delta_{3} \bigsqcup \Delta_{4}$, where

$$
\begin{aligned}
& \Delta_{1}=g_{1}^{-1} g_{3}^{-2} x_{0}\left(C_{3}\right) \\
& \Delta_{2}=g_{1}^{-1} g_{3}^{-1} x_{0}\left(C_{4}\right) \\
& \Delta_{3}=g_{1}^{-2} g_{2}^{2}\left(x_{0}\left(C_{3}\right) \bigcup x_{0}\left(C_{5}\right)\right) \\
& \Delta_{4}=g_{1}^{-2} g_{2} g_{3}^{-1} g_{1}^{-1}\left(x_{0}\left(C_{1}\right) \bigcup x_{0}\left(C_{2}\right)\right) .
\end{aligned}
$$

Therefore it is obvious that

$$
\begin{aligned}
G & =x_{0}\left(C_{3}\right) \bigsqcup\left(G \backslash x_{0}\left(C_{3}\right)\right) \\
& =g_{3} g_{1} \Delta_{2} \bigsqcup\left(G \backslash x_{0}\left(C_{3}\right)\right)
\end{aligned}
$$

and besides

$$
G=g_{3}^{2} g_{1} \Delta_{1} \bigsqcup g_{2}^{-2} g_{1}^{2} \Delta_{3} \bigsqcup g_{1} g_{3} g_{2}^{-1} g_{1}^{2} \Delta_{4}
$$

In other words, we have a paradoxical decomposition consisting of 5 pieces. Thus $\tau(G) \leq 5$. This number can be obtained from the above diagram by counting the paths from the beginning points to the end points and adding the result by 1 . The 
suitable part of $\Gamma(G, \mathfrak{g}, \mathcal{E})$ corresponding to this example is

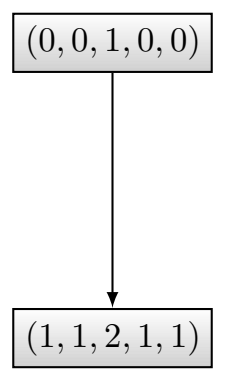

We have a very special case of Theorem 3.1 which eventuates a result for computing the Tarski number. We use the notations of section 3 .

Theorem 4.5. Suppose that $\sum_{i=1}^{p}\left(W_{i}-V_{i}\right)=(1,1, \ldots, 1)$ and there exists a permutation matrix $P_{\pi}$ such that the first $p-1$ rows of $P_{\pi} B-P_{\pi}^{+} A$ have nonnegative entries. Then $\tau(G) \leq 1+\left|A_{\pi(1)}\right|+\ell$.

Proof. The assumption says that for $1 \leq i \leq n, A_{\pi(i+1)} \subseteq B_{\pi(i)}$. Set $A_{\pi(n+1)}=\emptyset$, $h_{i}=\dot{g}_{\pi(1)}^{-1} \dot{g}_{\pi(2)}^{-1} \ldots \dot{g}_{\pi(i)}^{-1}$ and $R_{i}=h_{i}\left(\tilde{B}_{\pi(i)} \backslash \tilde{A}_{\pi(i+1)}\right)$, for $1 \leq i \leq p$. By induction on $p$ we see

$$
\tilde{A}_{\pi(1)}=\bigsqcup_{i=1}^{p} R_{i} .
$$

Since by assumption $\sum_{i=1}^{p}\left(W_{i}-V_{i}\right)=(1,1, \ldots, 1)$, the equations

$$
\bigsqcup_{C \in\left(B_{\pi(i-1)} \backslash A_{\pi(i)}\right)} x_{0}(C)=h_{i}^{-1} R_{i}, \quad(1 \leq i \leq p)
$$

define a paradoxical decomposition. On the other hand we have

$$
\begin{aligned}
\tau(G) & \leq\left|B_{\pi(p)}\right|+\left(\sum_{i=2}^{n}\left|B_{\pi(i-1)} \backslash A_{\pi(i)}\right|\right)+1 \\
& =\left(\sum_{i=1}^{n}\left|B_{\pi(i)}-A_{\pi(i)}\right|\right)+\left|A_{\pi(1)}\right|+1 \\
& =\ell+\left|A_{\pi(1)}\right|+1 .
\end{aligned}
$$

In the special case where $G$ does not contain the free group on two generators and $\ell=3$ and $\left|A_{\pi}(1)\right|=1$ we have $\tau(G)=5$.

Example 4.6. Let $\operatorname{Con}(\mathfrak{g}, \mathcal{E})=\{(1,2,2,2),(2,1,2,1),(2,2,1,1)\}$. Then $\tau(G) \leq 5$. A minimal diagram associated with $E q(\mathfrak{g}, \mathcal{E})$ is 


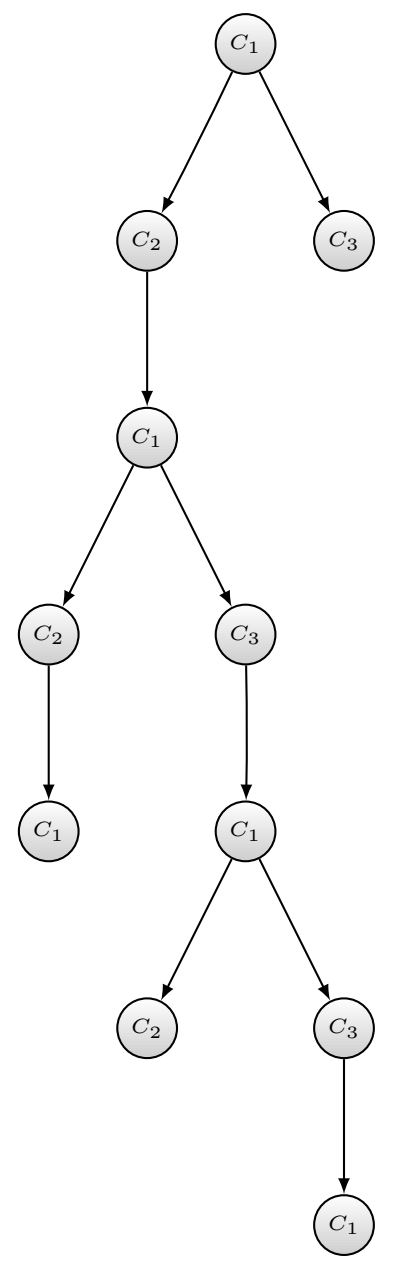

The number of paths from the top to the bottom is 4 . These paths are $\left[C_{1}, C_{3}\right],\left[C_{1}, C_{2}, C_{1}, C_{2}, C_{1}\right]$, $\left[C_{1}, C_{2}, C_{1}, C_{3}, C_{1}, C_{2}\right]$ and $\left[C_{1}, C_{2}, C_{1}, C_{3}, C_{1}, C_{3}, C_{1}\right]$. Therefore $\tau(G) \leq 4+1=5$.

Indeed according to (5) we have

$$
\begin{aligned}
& x_{0}\left(C_{1}\right)=g_{3} x_{0}\left(C_{2}\right) \cup g_{3} x_{0}\left(C_{3}\right) \\
& x_{0}\left(C_{2}\right)=g_{1}^{-1} x_{0}\left(C_{1}\right) \\
& x_{0}\left(C_{3}\right)=g_{2} x_{0}\left(C_{1}\right) .
\end{aligned}
$$

Define

$$
\begin{aligned}
& \Delta_{1}=g_{3} g_{1}^{-1} g_{3} g_{1}^{-1} g_{3} x_{0}\left(C_{2}\right), \\
& \Delta_{2}=g_{3} g_{1}^{-1} g_{3} g_{1}^{-1} g_{3} g_{2} x_{0}\left(C_{1},\right) \\
& \Delta_{3}=g_{3} g_{1}^{-1} g_{3} g_{2} x_{0}\left(C_{1}\right), \\
& \Delta_{4}=g_{3} x_{0}\left(C_{3}\right), \\
& \Delta_{5}=x_{0}\left(C_{2}\right) \cup x_{0}\left(C_{3}\right) .
\end{aligned}
$$


Then $x_{0}\left(C_{1}\right)=\bigsqcup_{i=1}^{4} \Delta_{i}$. So, $G=\bigsqcup_{i=1}^{5} \Delta_{i}$ and finally we have

$$
\begin{aligned}
& G=g_{2}^{-1} g_{3}^{-1} g_{1} g_{3}^{-1} \Delta_{3} \bigsqcup g_{3}^{-1} g_{1} g_{3}^{-1} g_{1} g_{3}^{-1} \Delta_{1} \bigsqcup g_{3}^{-1} \Delta_{4} \\
& G=g_{2}^{-1} g_{3}^{-1} g_{1} g_{3}^{-1} g_{1} g_{3}^{-1} \Delta_{2} \bigsqcup e \Delta_{5},
\end{aligned}
$$

where $e$ is the identity element of $G$. We have a complete paradoxical decomposition with five pieces. So, $\tau(G) \leq 5$.

Example 4.7. 8] Let $\mathbb{F}_{2}=\langle a, b\rangle$ be the free group with generators $a$ and $b$ and identity element $e$. Suppose that $\mathfrak{g}=(a, b)$ and $\mathcal{E}=\left\{E_{1}, E_{2}, E_{3}\right\}$, where

$E_{1}=\{x, \mathrm{x}$ is a reduced word starting with a $\}$,

$E_{2}=\{x, \mathrm{x}$ is a reduced word starting with $\mathrm{b}\}$ and

$E_{3}=\left\{x, \mathrm{x}\right.$ is a reduced word starting with $a^{-1}$ or $\left.b^{-1}\right\} \sqcup\{e\}$.

Then $\operatorname{Con}(\mathfrak{g}, \mathcal{E})=\left\{C_{1}, \ldots, C_{7}\right\}$, where $C_{1}=(1,1,2), C_{2}=(2,1,2), C_{3}=(3,1,2), C_{4}=$ $(3,2,2), C_{5}=(3,3,2), C_{6}=(3,1,3)$ and $C_{7}=(3,1,1)$. One can see that this example satisfies that condition of the pervious theorem. Consider the following configuration equations

$$
\sum_{x_{0}(C) \subseteq E_{1}} f_{C}=\sum_{x_{1}(C) \subseteq E_{1}} f_{C} \text { and } \sum_{x_{0}(C) \subseteq E_{2}} f_{C}=\sum_{x_{2}(C) \subseteq E_{2}} f_{C}
$$

i.e.

$$
f_{C_{1}}=f_{C_{1}}+f_{C_{2}}+f_{C_{3}}+f_{C_{6}}+f_{C_{7}} \text { and } f_{C_{2}}=f_{C_{1}}+f_{C_{2}}+f_{C_{3}}+f_{C_{4}}+f_{C_{5}},
$$

which imply that each $f_{C}$ is zero. The corresponding diagram is

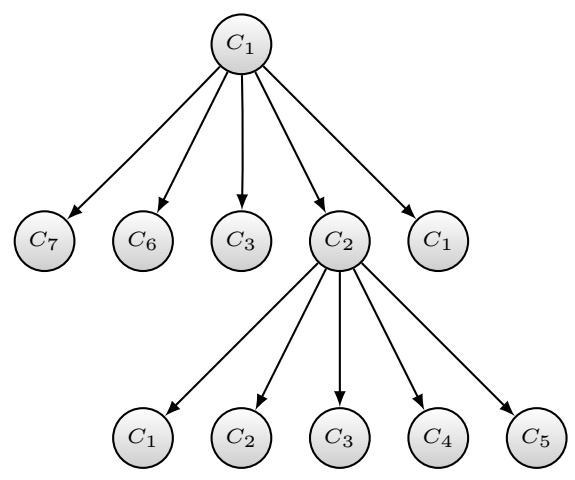

Put $A=a^{-1} x_{0}\left(C_{1}\right), B=b^{-1} a^{-1}\left[\cup_{i=1}^{5} x_{0}\left(C_{i}\right)\right], C=a^{-1}\left(x_{0}\left(C_{6}\right) \cup x_{0}\left(C_{7}\right)\right)$ and $M=\mathbb{F}_{2} \backslash x_{0}\left(C_{1}\right)$. Then we have the non-complete paradoxical decomposition

$$
\mathbb{F}_{2}=A \sqcup B \sqcup C \sqcup M \sqcup a^{-1} x_{0}\left(C_{3}\right)=e M \sqcup a A=a b B \sqcup a C .
$$

Hence $\tau\left(\mathbb{F}_{2}\right)=4$.

We now summarize what we have done for estimating the upper bound of the Tarski number of a group.

(1) Change the order of the configuration equations based on the permutation matrix in the definition of the normality condition. 
(2) Define the initial diagrams consisting of $p$ connected components.

(3) Make each set in the top of the above diagrams disjoint from the previous ones by borrowing their intersection from the sets in the bottom of the former diagrams.

(4) Glue these diagrams as it is explained in subsection 4.1.

(5) Find the configurations of the top sets of the initial diagrams in the bottom of the last gluing diagram.

(6) Count all the directed pats from top to the bottom. This number plus one is an upper bound for $\tau(G)$.

To get the best bound for $\tau(G)$ using this procedure, we borrow the maximum number of configurations that we can get from the former bottom sets (not only the intersections). It is applied in the examples.

Definition 4.8. Let $m, n \in \mathbb{N}$. A set of $(n+1)$-tuples $\mathcal{C}=\left\{\left(c_{0}^{i}, \ldots, c_{n}^{i}\right), \quad 1 \leq i \leq \ell\right\}$ with $1 \leq c_{j}^{i} \leq m$ and

$$
\cup_{j=1}^{n}\left\{c_{j}^{i}\right\}=\{1, \ldots, m\}, \quad(i=1, \ldots, \ell)
$$

is called a pre-configuration set if there exist a group $G$, a string $\mathfrak{g}$ of elements of $G$ and a partition $\mathcal{E}$ of $G$ such that $\operatorname{Con}(\mathfrak{g}, \mathcal{E})=\mathcal{C}$.

In 5 the authors give examples of groups with Tasrki numbers 5 and 6 . Now the question is whether we can construct such groups using configurations. Responding to this question depends on knowing that given well-behaved sets are pre-configuration ones. In particular

Question 4.9. Is $\mathcal{C}=\{(1,2,3,2),(1,3,1,3),(2,1,2,2),(3,3,1,2),(3,3,2,1)\}$ a preconfiguration set (see Example 4.4)?

\section{REFERENCES}

[1] A. Abdollahi, A. Rejali and G. A. Willis, Group properties characterized by configuration, Illinois J. Mathematics, 48 (2004) No. 3, 861-873.

[2] A. Abdollahi, A. Rejali and A. Yousofzadeh, Configuration of nilpotent groups and isomorphism, J. Algebra Appl, 8, No. 3 (2009) 339-350.

[3] T. G. Ceccherini-Silberstein, R. I. Grigorchuk and P. de la Harpe, Amenability and paradoxical decompositions for pseudogroups and for discrete metric spaces, Proc. Steklov. Inst. Math. 224 (1999), 57-97.

[4] G. B. Dantzig and M. N. Thapa, Linear Programming 2: Theory and Extensions. SpringerVerlag, New York, 2003.

[5] M. Ershov, G. Golan and M. Sapir, The Tarski numbers of groups, Adv. Math. 284 (2015) $21-53$.

[6] A. 1. T. Paterson, Amenability Mathematical Surveyes and Monographs., 29, American Mathematical Society, Providence, RI, 1988.

[7] A. Rejali and A. Yousofzadeh, Configuration of groups and paradoxical decompositions, Bull. Belg. Math. Soc. Simon Stevin 18 (2011) 157-172.

[8] J. M. Rosenblatt and G. A. Willis, Weak convergence is not strong for amenable groups, Canad. math. Bull, 44 (2001) No 2, 231-241.

[9] M. Sapir, Combinatorial algebra: Syntax and Semantics. Springer International Publishing, Suitzerland, 2014.

[10] S. Wagon, The Banach-Tarski Paradox., vol. 24 of Encyclopedia of Mathematics and its Applications, Cambridge University Press, Cambridge-New York, 1985.

[11] A. Yousofzadeh, A. Tavakoli and A. Rejali, On configuration graph and paradoxical decomposition,J. Algebra. Appl, 13, No. 2 (2014), 1350086 (11 pages).

E-mail address: ayousofzade@yahoo.com 